\title{
The $\log \log$ growth of channel capacity for nondispersive nonlinear optical fiber channel in intermediate power range
}

\author{
I. S. Terekhov ${ }^{1,2, *}$ A. V. Reznichenko, ${ }^{1,2, \dagger}$ Ya. A. Kharkov ${ }^{3, \ddagger}$ and S. K. Turitsyn ${ }^{4,2, \S}$ \\ ${ }^{1}$ Budker Institute of Nuclear Physics of Siberian Branch Russian Academy of Sciences, Novosibirsk, 630090 Russia \\ ${ }^{2}$ Novosibirsk State University, Novosibirsk, 630090 Russia \\ ${ }^{3}$ School of Physics, University of New South Wales, Sydney 2052, Australia \\ ${ }^{4}$ Aston Institute of Photonics Technologies, Aston University, Aston Triangle, Birmingham, B4 7ET, UK
}

\begin{abstract}
We consider a model nondispersive nonlinear optical fiber channel with an additive Gaussian noise. Using Feynman path-integral technique we find the optimal input signal distribution maximizing the channel's per-sample mutual information at large signal-to-noise ratio in the intermediate power range. The optimal input signal distribution allows us to improve previously known estimates for the channel capacity. We calculate the output signal entropy, conditional entropy, and per-sample mutual information for Gaussian, half-Gaussian and modified Gaussian input signal distributions. We demonstrate that in the intermediate power range the capacity (the per-sample mutual information for the optimal input signal distribution) is greater than the per-sample mutual information for half-Gaussian input signal distribution considered previously as the optimal one. We also show that the capacity grows as $\log \log P$ in the intermediate power range, where $P$ is the signal power.
\end{abstract}

PACS numbers:

\section{INTRODUCTION.}

Information theory is an interdisciplinary science, that has a broad range of applications in such fields as complex systems, statistical physics, computer science, data compression, engineering, genetics and etc. One of the most important and practically significant applications of information theory is the information transmission in communication systems. Due to a constant demand in the increase of the communication speed and quality finding a way to maximize amount of information transmitted through a noisy information channel has a tremendous value for modern communication technology. The problem of an informational capacity of a linear channel with a Gaussian noise has been first considered by Shannon in his seminal work [1]. Shannon introduced the channel capacity as the maximal amount of information per symbol that can be transmitted via the noisy channel, and demonstrated that the capacity $C$ can be expressed as:

$$
C \propto \log _{2}(1+\mathrm{SNR}),
$$

where $\mathrm{SNR}=P / N$ is the signal-to-noise power ratio, $P$ is the signal power, and $N$ is the noise power. This, in particular, means that for the fixed noise power $N$ one has to increase the signal power $P$ in order to increase the capacity.

The interest in nonlinear communication channels has been growing since the beginning of the 2000's when fiber optical communication systems had to extend both bandwidth and the system's reach, that required an increase

\footnotetext{
*Electronic address:i.s.terekhov@gmail.com

$\dagger$ Electronic address: a.v.reznichenko@inp.nsk.su

¥Electronic address:y.kharkov@gmail.com

$\S$ Electronic address:s.k.turitsyn@aston.ac.uk
}

of the input optical power. The Kerr nonlinearity in optical fibres strongly affects the information capacity, that have been studied both analytically and numerically in numerous papers, see e.g. [2-9] and references therein. The information transmission in a simplified model of a nondispersive nonlinear optical fiber channel was considered, e.g. in Refs. [10-14]. The study of nonlinear communication channels where the transmission is affected by the signal power is a difficult problem, especially at large SNR [6]. The analysis of the capacity of these channels is technically challenging and new techniques and methods are highly desirable to advance these studies $[3,13,15-17]$. In order to address the problem of information capacity we consider a simplified model of a nonlinear channel with zero dispersion. The methods developed for and tested on such model channel might be useful for more complex nonlinear fiber communication problems. We introduce here a new approach to the calculation of the conditional probability density function via the path-integral technique and demonstrate its application using the considered model channel as a particular example.

The channel capacity $C$ is defined as the maximum of the mutual information $I_{P_{X}[X]}$ with respect to the probability density function $P_{X}[X]$ of the input signal $X$ :

$$
C=\max _{P_{X}[X]} I_{P_{X}[X]}
$$

where the maximum value of $I_{P_{X}[X]}$ should be found subject to the condition of fixed average signal power:

$$
P=\int \mathcal{D} X|X|^{2} P_{X}[X]
$$

The mutual information of a memoryless channel is defined in terms of the output signal entropy $H[Y]$ and the conditional entropy $H[Y \mid X]$ :

$$
I_{P_{X}[X]}=H[Y]-H[Y \mid X],
$$


with

$$
\begin{aligned}
H[Y \mid X] & =-\int \mathcal{D} X \mathcal{D} Y P_{X}[X] P[Y \mid X] \log P[Y \mid X],(5) \\
H[Y] & =-\int \mathcal{D} Y P_{\text {out }}[Y] \log P_{\text {out }}[Y] \\
P_{\text {out }}[Y] & =\int \mathcal{D} X P_{X}[X] P[Y \mid X]
\end{aligned}
$$

where $P[Y \mid X]$ is the conditional probability density function (PDF) for an output signal $Y$ when the input signal is $X$, and $P_{\text {out }}[Y]$ is the PDF for an output signal $Y$. The measure $\mathcal{D} Y$ is defined as $\int \mathcal{D} Y P[Y \mid X]=1$, and $\mathcal{D} X$ is defined as $\int \mathcal{D} X P_{X}[X]=1$. The capacity (2), as defined by (4)-(7), is measured in units of $(\log 2)^{-1}$ bits per symbol (also known as nats per symbol). The input and output signals are functions of time where the signal's spectrum is restricted to a given bandwidth. In general, a sampling of the temporal signal should be introduced to define a discrete-time memoryless channel, however, here we consider only per-sample quantities.

The channel's mutual information (4) depends on the probability distribution $P_{X}[X]$ of the input signal. The input signal PDF maximizing the channel's per-sample mutual information is called "capacity-approaching" or "optimal" PDF $P_{X}^{o p t}[X]$. Obviously, the problem of finding the optimal PDF of the input signal for nonlinear optical channels is of a great practical importance.

In the previous studies of nondispersive nonlinear optical channels (e.g. [11], [13], [14]) the Gaussian and halfGaussian input signal PDF's were used as trial functions in order to put low bound constraint on the channel capacity, or to provide an asymptotic estimate of the capacity in the regime of a large SNR. The authors of [14] argued, that the half-Gaussian PDF which we denote as $P_{X}^{(1)}[X]$

$$
P_{X}^{(1)}[X]=\frac{\exp \left\{-|X|^{2} /(2 P)\right\}}{\pi|X|(2 \pi P)^{1 / 2}},
$$

provides the best approximation for the "capacityapproaching" input signal distribution at a large SNR. In the present paper by solving a variational problem we show that it is not the case. We find a true optimal distribution $P_{X}^{o p t}[X]$ (which in fact is different from half-Gaussian distribution) in the regime of large SNR for intermediate power range. We explicitly show, that in this regime the mutual information (4) for our optimal input signal PDF is greater than the mutual information for the Gaussian and half-Gaussian input signal distributions.

The estimates for the capacity of nonlinear fiber channels with a zero dispersion and an additive white Gaussian noise in the regime of large SNR were obtained in Refs. [13], [14]. The lower bound for capacity of the channel, based on a trial Gaussian input signal PDF, reads [13]:

$C \geq \frac{\log (\mathrm{SNR})}{2}+\frac{1+\gamma_{E}-\log (4 \pi)}{2}+\mathcal{O}\left(\frac{\log (\mathrm{SNR})}{\mathrm{SNR}}\right)$ where $\gamma_{E} \approx 0.5772$ is the Euler constant. Note that the second term on the right-hand side of Eq. (9) was presented as $\mathcal{O}(1)$ in Ref. [13] but can be easily calculated using Eqs. (23) and (24) of Ref. [13]. The physical meaning of the pre-logarithmic factor $1 / 2$ in Eq. (9) is that the signal's phase does not carry information in the high power regime, see Ref. [14]. In fact, when the signal power is sufficiently large, i.e. $P \gtrsim\left(N \gamma^{2} L^{2}\right)^{-1}$, the signal-dependent phase occupies the entire phase interval $[0,2 \pi]$ due to self phase modulation and, as a result, the phase does not transfer information. Here we denote $\gamma$ as the Kerr nonlinearity coefficient and $L$ as the fiber link length. Capacity estimates in the intermediate power range $N \ll P \ll 6 \pi^{2}\left(N \gamma^{2} L^{2}\right)^{-1}$ are presented in Ref. [14]. For such a power $P$ the following estimate of the lower bound for the capacity, based on the half-Gaussian input signal PDF, was derived [14]:

$$
\begin{aligned}
C \geq & -\log (\gamma N L)+ \\
& \frac{\gamma_{E}-1+\log (3 \pi)}{2}+\mathcal{O}(1 / \sqrt{\mathrm{SNR}}),
\end{aligned}
$$

where instead of $\mathcal{O}(1 / \sqrt{\mathrm{SNR}})$ the authors presented the explicit function of the parameter SNR which decreases at large SNR, see Eq. (40) in [14]. However, the authors of [14] did not take into account the $1 / \sqrt{\mathrm{SNR}}$ corrections in the output signal entropy $H[Y]$, therefore, using these explicit functions in the capacity inequality is beyond the calculation accuracy. It also means that the result Eq. (40) of Ref. [14] is not a lower bound on the capacity. It is worth mentioning that in the inequality (10) there is an additive term $\log 2$ missing. Also Eq. (40) in [14] does not recover the Shannon limit log SNR as $\gamma \rightarrow 0$. Moreover, it is strange that the capacity estimate goes to infinity when $\gamma$ tends to zero. So there are obvious flaws in the inequality (10). Therefore, to understand the behavior of the capacity in the intermediate power range the additional study is necessary.

The analytical expression for the conditional probability density function of the channel was obtained in the complex form of an infinite series [10, 13, 14] within the Martin-Siggia-Rose formalism based on quantum field theory methods [18]. In the present paper we adopt the Martin-Siggia-Rose formalism and develop a new method for the approximate computation of the conditional probability density function $P[Y \mid X]$. Using this method we obtain the simple analytical expression for the function $P[Y \mid X]$ in the leading and next-to-leading order in the parameter $1 / \sqrt{\mathrm{SNR}}$ for the intermediate power regime

$$
N \ll P \ll\left(N \gamma^{2} L^{2}\right)^{-1}
$$

Our method allows us first to derive the analytical expression for the mutual information and then the optimal input signal distribution $P_{X}^{o p t}[X]$ which is different from the half-Gaussian. 
In [17] a method to calculate the conditional PDF for a nonlinear optical fiber channel with nonzero dispersion in the large SNR limit was introduced. Here we illustrate this general approach in the application to a simple nondispersive nonlinear optical fiber channel as considered in $[10,13,14]$. Since the channel is dispersionless, the temporal signal waveform does not change during propagation (note, however, that the signal bandwidth will grow due to the fiber nonlinearity and signal modulation). Therefore, instead of considering the evolution of $\psi(z, t)$ we can consider a set of independent scalar channels $[10,14]$ (per-sample channels) governed by the following model:

$$
\partial_{z} \psi(z)-i \gamma|\psi(z)|^{2} \psi(z)=\eta(z),
$$

where $\psi(z)$ is the signal function that is assumed to obey the boundary conditions $\psi(0)=X, \psi(L)=Y$. The noise $\eta(z)$ has zero mean $\langle\eta(z)\rangle_{\eta}=0$ and a correlation function $\left\langle\eta(z) \bar{\eta}\left(z^{\prime}\right)\right\rangle_{\eta}=Q \delta\left(z-z^{\prime}\right)$, so that the $\mathrm{SNR}=P / Q L$, where $P$ and $N=Q L$ are the per-sample signal power and the per-sample noise power, respectively. The connection between the model (12) and the conventional information-theoretic presentation in the form of an explicit input-output probabilistic model and the appropriate sampling has been discussed in detail in $[10,13,14]$. In order to illustrate our method we calculate the conditional probability density function, the conditional entropy (5), the output signal entropy (6), and the mutual information (4) for the per-sample channel. Solving a variational problem for the mutual information we find the optimal input signal distribution $P_{X}[X]$ maximizing the mutual information in the leading order in $1 / \mathrm{SNR}$.

The paper is organized as follows. In Section II we develop a "quasi-classical" method for the calculation of the conditional PDF $P[Y \mid X]$ for arbitrary nonlinearity in the intermediate power range (11) in the leading and next-to-leading order in $1 / \sqrt{\mathrm{SNR}}$. We find a simple representation for $P[Y \mid X]$ in this case. This allows us to calculate the output signal distribution $P_{\text {out }}[Y]$. The optimal signal distribution $P_{X}^{o p t}[X]$ is found in Section III. Section IV is focused on the calculation and the comparison of the mutual information for various input signal distributions. We discuss our results in Section V.

\section{THE CONDITIONAL PDF $P[Y \mid X]$ AND OUTPUT SIGNAL PDF $P_{\text {out }}[Y]$ AT LARGE SNR}

\section{A. "Quasi-classical" method for the conditional PDF $P[Y \mid X]$ calculation}

The conditional probability density function can be written via the path-integral form $[13,18,19]$ in a retarded discretization scheme, see e.g. Supplemental Materials of Ref. [17]

$$
P[Y \mid X]=\int_{\psi(0)=X}^{\psi(L)=Y} \mathcal{D} \psi \exp \left\{-\frac{S[\psi]}{Q}\right\},
$$

and can be reduced to the quasi-classical form, see Ref. [19]:

$$
P[Y \mid X]=e^{-\frac{S\left[\Psi_{c l}(z)\right]}{Q}} \int_{\tilde{\psi}(0)=0} \mathcal{D} \tilde{\psi} e^{-\frac{S\left[\Psi_{c l}(z)+\tilde{\psi}(z)\right]-S\left[\Psi_{c l}(z)\right]}{Q}},
$$

where the effective action $S[\psi]=\left.\left.\int_{0}^{L} d z\left|\partial_{z} \psi-i \gamma\right| \psi\right|^{2} \psi\right|^{2}$, and the function $\Psi_{c l}(z)$ is the "classical" solution of the equation $\delta S\left[\Psi_{c l}\right]=0$, where $\delta S$ is the variation of our action $S[\psi]$. The equation $\delta S\left[\Psi_{c l}\right]=0$ (Euler-Lagrange equation) has the form

$$
\frac{d^{2} \Psi_{c l}}{d z^{2}}-4 i \gamma\left|\Psi_{c l}\right|^{2} \frac{d \Psi_{c l}}{d z}-3 \gamma^{2}\left|\Psi_{c l}\right|^{4} \Psi_{c l}=0
$$

with the boundary conditions $\Psi_{c l}(0)=X, \Psi_{c l}(L)=Y$.

In order to find $P[Y \mid X]$ one should calculate the exponent $e^{-\frac{S\left[\Psi_{c l}(z)\right]}{Q}}$ and the path-integral in Eq. (14). First, we evaluate the exponent. To find it we have to calculate the function $\Psi_{c l}(z)$ and then the action $S\left[\Psi_{c l}(z)\right]$. We found the general solution $\Psi_{c l}(z)$ of (15) implicitly through the boundary conditions, see Eqs. (A8)-(A12), and Eq. (A14) in Appendix A. This form of the solution is inconvenient for further calculations. Therefore we adopt a different approach and find the solution in the leading and next-to-leading order in $1 / \sqrt{\mathrm{SNR}}$, linearizing Eq. (15) in the vicinity of the solution $\Psi_{0}(z)$. Here $\Psi_{0}(z)$ is the solution of the equation (12) with zero noise and with the boundary condition $\Psi_{0}(0)=X=\rho e^{i \phi^{(X)}}$. The function $\Psi_{0}(z)$ reads

$$
\Psi_{0}(z)=\rho \exp \left\{i \mu \frac{z}{L}+i \phi^{(X)}\right\},
$$

where $\mu=\gamma L \rho^{2}=\gamma L|X|^{2}$. Note that this solution satisfies only the input boundary condition $\Psi_{0}(0)=X=$ $\rho e^{i \phi^{(X)}}$, and it is the solution of Eq. (15) as well. Therefore, we look for the solution of Eq. (15) in the following form

$$
\Psi_{c l}(z)=(\rho+\varkappa(z)) \exp \left\{i \mu \frac{z}{L}+i \phi^{(X)}\right\},
$$

where the function $\varkappa(z)$ is assumed to be small: $|\varkappa(z)| \ll$ $\rho$. In a general case, the ratio $|\varkappa(z)| / \rho$ is not necessarily small and it depends on the output boundary condition $\varkappa(L)$. However, the configurations of $\varkappa(z)$ at which $\Psi_{c l}(z)$ significantly deviates from $\Psi_{0}(z)(|\varkappa(z)| \sim$ $\rho)$ are statistically irrelevant. Indeed, the expansion $S\left[\Psi_{0}(z)+\delta \Psi(z)\right] \propto \varkappa^{2}(z)$ starts from the quadratic term at small $\varkappa(z)$, since the action achieves an extremum (the absolute minimum $\left.S\left[\Psi_{0}(z)\right]=0\right)$ on the solution $\Psi_{0}(z)$. Thus the exponent $e^{-\frac{S\left[\Psi_{c l}(z)\right]}{Q}}$ and, as a result, the conditional PDF $P[Y \mid X]$ vanishes exponentially when the typical $\varkappa(z)$ is much greater than $\sqrt{Q L}$.

Substituting Eq. (17) into Eq. (15) and retaining only terms linear in $\varkappa(z) / \rho$, we obtain the following equation 
which is still exact in the non-linearity parameter $\mu$ :

$$
\frac{d^{2} \varkappa}{d z^{2}}-2 i \frac{\mu}{L} \frac{d \varkappa}{d z}-4 \frac{\mu^{2}}{L^{2}} \operatorname{Re}[\varkappa]=0 .
$$

The boundary conditions for the function $\varkappa(z)$ read

$$
\varkappa(0)=0, \varkappa(L)=Y e^{-i \phi^{(X)}-i \mu}-\rho \equiv x_{0}+i y_{0},
$$

where $x_{0}=\operatorname{Re}\{\varkappa(L)\}$ and $y_{0}=\operatorname{Im}\{\varkappa(L)\}$. The solution of the linearized boundary problem (18), (19) reads

$$
\begin{aligned}
& \operatorname{Re}[\varkappa(z)]=\left(\mu \frac{\mu x_{0}-y_{0}}{1+\mu^{2} / 3} \frac{z}{L}+\frac{\left(1-2 \mu^{2} / 3\right) x_{0}+\mu y_{0}}{1+\mu^{2} / 3}\right) \frac{z}{L}, \\
& \operatorname{Im}[\varkappa(z)]=\left\{\frac{\mu x_{0}-y_{0}}{1+\mu^{2} / 3}\left(\frac{2 \mu^{2} z^{2}}{3 L^{2}}-1\right)+\right. \\
& \left.\mu \frac{\left(1-2 \mu^{2} / 3\right) x_{0}+\mu y_{0}}{1+\mu^{2} / 3} \frac{z}{L}\right\} \frac{z}{L} .
\end{aligned}
$$

After substitution of the solution Eq. (20) in the action we obtain

$$
\begin{aligned}
& \frac{1}{Q} S\left[\Psi_{c l}(z)\right]=\frac{1}{Q} S\left[(\rho+\varkappa(z)) \exp \left\{i \mu \frac{z}{L}+i \phi^{(X)}\right\}\right] \approx \\
& \frac{1}{Q} \int_{0}^{L} d z\left|\partial_{z} \varkappa-2 i \frac{\mu}{L} \operatorname{Re}[\varkappa]\right|^{2}= \\
& \frac{\left(1+4 \mu^{2} / 3\right) x_{0}^{2}-2 \mu x_{0} y_{0}+y_{0}^{2}}{Q L\left(1+\mu^{2} / 3\right)} .
\end{aligned}
$$

Note that here we retain only the terms quadratic in $\varkappa$. However, it is straightforward to calculate the next correction to the action (21) which is of the order of $1 / \sqrt{\mathrm{SNR}}$, see details in Appendix A. A regular perturbative expansion for $\varkappa(z)$ in powers of $1 / \sqrt{\mathrm{SNR}}$ can be obtained using the exact equation for the function $\varkappa(z)$, see Eq. (A19) in Appendix A.

The next step in evaluation of the conditional probability $P[Y \mid X]$ is the calculation of the path-integral in Eq. (14). In order to calculate the path-integral in the leading $1 / \sqrt{\mathrm{SNR}}$ order we retain only quadratic in $\tilde{\psi}$ terms in the integrand. Any extra power of $\tilde{\psi}$ or $\varkappa$ is suppressed by the multiplicative parameter $\sqrt{Q L}$, because at small $Q$ the main contribution to the path-integral comes from $\tilde{\psi} \sim \sqrt{Q L}$. Moreover, as soon as we calculate the path-integral in the leading order in $Q$, we can substitute $\Psi_{0}(z)$ for $\Psi_{c l}(z)$ in the action difference $S\left[\Psi_{c l}(z)+\tilde{\psi}(z)\right]-S\left[\Psi_{c l}(z)\right]$. To find $P[Y \mid X]$ in the nextto-leading order in $1 / \sqrt{\mathrm{SNR}}$ we should keep both $\varkappa(z)$ in $\Psi_{c l}(z)$ and higher powers of $\tilde{\psi}$ in the action difference. Details of the path-integral calculation in the leading and next-to-leading order in $1 / \sqrt{\mathrm{SNR}}$ are presented in Appendix B. Taking into account the expression for the action (A28) and the result of the path-integral calculation (B22) we obtain the final result for $P[Y \mid X]$ with the accuracy of corrections proportional to $Q L$ :

$$
\begin{aligned}
& P[Y \mid X]=\frac{\exp \left\{-\frac{\left(1+4 \mu^{2} / 3\right) x_{0}^{2}-2 \mu x_{0} y_{0}+y_{0}^{2}}{Q L\left(1+\mu^{2} / 3\right)}\right\}}{\pi Q L \sqrt{1+\mu^{2} / 3}}\left(1-\frac{\mu / \rho}{15\left(1+\mu^{2} / 3\right)^{2}}\left(\mu\left(15+\mu^{2}\right) x_{0}-2\left(5-\mu^{2} / 3\right) y_{0}\right)-\right. \\
& \frac{\mu / \rho}{135 Q L\left(1+\mu^{2} / 3\right)^{3}}\left\{\mu\left(4 \mu^{4}+15 \mu^{2}+225\right) x_{0}^{3}+\left(23 \mu^{4}+255 \mu^{2}-90\right) x_{0}^{2} y_{0}+\mu\left(20 \mu^{4}+117 \mu^{2}-45\right) x_{0} y_{0}^{2}-\right. \\
& \left.\left.3\left(5 \mu^{4}+33 \mu^{2}+30\right) y_{0}^{3}\right\}\right)
\end{aligned}
$$

where $x_{0}$ and $y_{0}$ are the functions of $X$ and $Y$ defined in (19). The corrections to $P[Y \mid X]$ proportional to $Q L$ can be found in our paper [20]. Note that the conditional PDF $P[Y \mid X]$ was already derived in $[13]$ in the form of an infinite series. Our result (22) for the function $P[Y \mid X]$ is the analytic summation of this series in the intermediate power range

$$
Q L \ll P \ll\left(Q L^{3} \gamma^{2}\right)^{-1} .
$$

The left inequality in Eq. (23) comes from the condition of large SNR, SNR 》1. The right inequality in Eq. (23) is the condition for the path-integral corrections (of order of $\gamma^{2} L^{3} Q|X|^{2}$ ) to be small: see Eq. (B2) in Appendix B, and Ref. [20]. One can show that the normalization condition $\int D Y P[Y \mid X]=1$ is fulfilled. Also one can check that the distribution (22) obeys the following important property

$$
\lim _{Q \rightarrow 0} P[Y \mid X]=\delta\left(Y-\Psi_{0}(L)\right) .
$$

The expression (24) is nothing else, but the deterministic limit of $P[Y \mid X]$ in the absence of noise. Also Eq. (22) has the correct limit for the linear channel $(\gamma \rightarrow 0)$ :

$$
P^{(0)}[Y \mid X]=\frac{e^{-|Y-X|^{2} / Q L}}{\pi Q L},
$$


that is nothing else but the conditional PDF for the linear nondispersive channel with Gaussian noise.

\section{B. Output signal PDF $P_{\text {out }}[Y]$}

Now we proceed to the calculation of the probability density function of the output signal $P_{\text {out }}[Y]$. Let us consider the integral, see Eq. (7),

$$
P_{\text {out }}[Y]=\int \mathcal{D} X P[Y \mid X] P_{X}[X]
$$

where the function $P_{X}[X]$ is a smooth function that changes on a scale $|X|^{2} \sim P$ which is much greater than $Q L$. In this case we can calculate the integral (26) up to terms proportional to the noise power $Q L$ by Laplace's method [21], see Appendix C. The result has the form:

$$
P_{\text {out }}[Y]=\int \mathcal{D} X P[Y \mid X] P_{X}[X]=P_{X}\left[Y e^{-i \gamma|Y|^{2} L}\right] .
$$

This result (27) can be obtained without calculations from the following reasoning. The function $P[Y \mid X]$, see Eq. (22), varies on a scale of order $Q L$ which is much less than the scale of $P_{X}[X]$ (the function $P[Y \mid X]$ is essentially narrower than the function $\left.P_{X}[X]\right)$. Also $P[Y \mid X]$ has the delta-function limit (24) and therefore in the integral (26) it can be replaced with the delta-function. Note that to obtain the result (27) we do not require the limit $Q \rightarrow 0$ but only the relation between the scales $P$ and $Q L$ to be satisfied. For the case of the distribution $P_{X}[X]$ which depends only on $|X|$ we have $P_{\text {out }}[Y]=P_{X}[|Y|]$.

For the case when $P_{X}[X]$ depends only on $|X|$ we can obtain $P_{\text {out }}[Y]$ in all orders in $Q L$. In the remainder of this Section we consider this case. To obtain $P_{\text {out }}[Y]$ we can use the $P[Y \mid X]$ found in Ref. [13], see Eqs. (11)-(13) therein. In this case $P_{\text {out }}[Y]$ is a function of $|Y|=\rho^{\prime}$

$$
P_{\text {out }}\left[\rho^{\prime}\right]=\frac{2 e^{-\frac{\rho^{\prime 2}}{Q L}}}{Q L} \int_{0}^{\infty} d \rho \rho e^{-\frac{\rho^{2}}{Q L}} I_{0}\left(\frac{2 \rho \rho^{\prime}}{Q L}\right) P_{X}[\rho],
$$

where $I_{0}(z)$ is the modified Bessel function of the first kind. Using this representation we can obtain the simple relation for $P_{\text {out }}\left[\rho^{\prime}\right]$ calculation in the perturbation theory in $Q L$. To this end we perform the zero order Hankel transformation [21]:

$$
\hat{P}[k]=\int_{0}^{\infty} d \rho \rho J_{0}(k \rho) P_{X}[\rho] .
$$

of both sides of Eq. (28), then we use the standard integral [22] with Bessel and modified Bessel functions

$$
\int_{0}^{\infty} d z z e^{-p z^{2}} J_{\nu}(b z) I_{\nu}(c z)=\frac{1}{2 p} J_{\nu}\left(\frac{b c}{2 p}\right) e^{\frac{c^{2}-b^{2}}{4 p}},
$$

and arrive at the simple relation between the Hankel images

$$
\hat{P}_{\text {out }}[k]=e^{-k^{2} \frac{Q L}{4}} \hat{P}[k] .
$$

Performing the inverse Hankel transformation

$$
P_{X}[\rho]=\int_{0}^{\infty} d k k J_{0}(k \rho) \hat{P}[k]
$$

we obtain

$$
P_{\text {out }}[\rho]=e^{\frac{Q L}{4} \Delta_{\rho}} P_{X}[\rho],
$$

where $\Delta_{\rho}=\frac{d^{2}}{d \rho^{2}}+\frac{1}{\rho} \frac{d}{d \rho}$ is the two-dimensional radial Laplace operator. From the relation (32) the problem of finding $(Q L)^{n}$ corrections to $P_{\text {out }}[\rho]$ reduces to the exponent expansion and straightforward calculations of the action of the differential operator $\Delta_{\rho}^{n}$ on $P_{X}[\rho]$.

Let us consider the widely used example of the modified Gaussian distribution

$$
P_{X}^{(\beta)}[\rho]=\frac{\exp \left\{-\beta \rho^{2} /(2 P)\right\} \rho^{\beta-2}}{\pi \Gamma(\beta / 2)(2 P / \beta)^{\beta / 2}} .
$$

For $\beta>0$ the distribution $P_{X}^{(\beta)}[\rho]$ is normalized to unity, $2 \pi \int_{0}^{\infty} d \rho \rho P_{X}^{(\beta)}[\rho]=1$, and has the average power $P$, $2 \pi \int_{0}^{\infty} d \rho \rho^{3} P_{X}^{(\beta)}[\rho]=P$. The distribution $P_{X}^{(\beta)}[X]$ generalizes the half-Gaussian distribution (8) for $\beta=1$ and the Gaussian for $\beta=2$ :

$$
P_{X}^{(2)}[X]=\frac{1}{\pi P} e^{-|X|^{2} / P}
$$

Inserting (33) into Eq. (28) we obtain a standard integral which can be found in [22]. The result for the output signal PDF has the form:

$$
\begin{aligned}
P_{\text {out }}^{(\beta)}[Y]= & { }_{1} F_{1}\left(\frac{\beta}{2} ; 1 ; \frac{|Y|^{2} 2 P}{Q L(2 P+\beta Q L)}\right) \times \\
& \frac{\exp \left\{-|Y|^{2} / Q L\right\}}{\pi Q L}\left(\frac{\beta Q L}{2 P+\beta Q L}\right)^{\beta / 2}
\end{aligned}
$$

where ${ }_{1} F_{1}\left(\frac{\beta}{2} ; 1 ; z\right)$ is the confluent hypergeometric function that reduces to $e^{z}$ for the Gaussian case and to $e^{z / 2} I_{0}(z / 2)$ for the half-Gaussian case:

$$
\begin{aligned}
P_{\text {out }}^{(2)}[Y]=\frac{1}{\pi(P+Q L)} \exp \left\{-\frac{|Y|^{2}}{P+Q L}\right\}, & \\
P_{\text {out }}^{(1)}[Y]= & \frac{1}{\pi \sqrt{Q L(2 P+Q L)}} I_{0}\left(\frac{|Y|^{2} P}{Q L(2 P+Q L)}\right) \times \\
& \exp \left\{-\frac{|Y|^{2}(P+Q L)}{Q L(2 P+Q L)}\right\} .
\end{aligned}
$$

Note that the result for $P_{\text {out }}^{(1)}[Y]$ in Ref. [14], see Eq. (38) therein, for the half-Gaussian distribution is incorrect. 
We can reproduce the general result of Eq. (27) by considering Eq. (37) in the case $Q L \ll|Y|^{2} \sim P$. For the case one can obtain:

$$
P_{\text {out }}^{(1)}[Y] \approx P_{X}^{(1)}[|Y|]
$$

with accuracy of the terms proportional to $Q L$. The result (38) coincides with Eq. (27).

\section{OPTIMAL INPUT SIGNAL DISTRIBUTION AT LARGE SNR}

The optimal input signal distribution at large SNR can be found calculating the mutual information (4) and then maximizing the result with respect to the input signal distribution function $P_{X}[X]$. Let us start from the calculation of the output signal entropy $H[Y]$, see Eq. (6), at large SNR.
When the parameter SNR $\gg 1$ we can substitute $P_{X}\left[Y \exp \left\{-i \gamma|Y|^{2} L\right\}\right]$ instead of $P_{\text {out }}[Y]$ due to the relation (27):

$$
H[Y]=-\int_{0}^{2 \pi} d \phi \int_{0}^{\infty} d \rho^{\prime} \rho^{\prime} P_{X}\left[\rho^{\prime} e^{i \phi}\right] \log P_{X}\left[\rho^{\prime} e^{i \phi}\right]
$$

In order to obtain Eq. (39) we have performed the change of the integration variable $\phi=\phi^{(Y)}+\gamma|Y|^{2} L$. One can see that the output signal entropy (39) coincides with the input signal entropy $H[X]$ up to terms proportional to $Q L$.

The conditional entropy $H[Y \mid X]$ can be calculated by substitution of $P[Y \mid X]$ in the form of Eq. (22) into Eq. (5). After the substitution we change the integration variables $\mathcal{D} Y \equiv d \operatorname{Re} Y d \operatorname{Im} Y$ to $d x_{0} d y_{0}$. Then we perform integration over $x_{0}, y_{0}$ and obtain

$$
H[Y \mid X]=1+\log (\pi Q L)+\frac{1}{2} \int_{0}^{2 \pi} d \phi^{(X)} \int_{0}^{\infty} d \rho \rho P_{X}\left[\rho e^{i \phi^{(X)}}\right] \log \left(1+\frac{\gamma^{2} L^{2}}{3} \rho^{4}\right)
$$

where the first two terms in the r.h.s. of the equation come from the Gaussian type integrals over $\mathcal{D} Y$ in the conditional entropy definition (5) and the normalization factor $\pi Q L$ in Eq. (22). The third term in Eq. (40) comes from the normalization factor $\sqrt{1+\mu^{2} / 3}$, see Eq. (22). Note that there are no terms which are proportional to $\sqrt{Q L}$ in Eqs. (39) and (40). Indeed, the integrals with the odd powers of $x_{0}$ and $y_{0}$ vanish when integrating over $x_{0}, y_{0}$ in Eq. (5) for $H[Y \mid X]$.

To find the optimal distribution $P_{X}^{o p t}[X]$ normalized to unity and having a fixed average power $P$ one should solve the variational problem for the functional $J\left[P_{X}, \lambda_{1}, \lambda_{2}\right]$

$$
J\left[P_{X}, \lambda_{1}, \lambda_{2}\right]=H[Y]-H[Y \mid X]-\lambda_{1}\left(\int \mathcal{D} X P_{X}[X]-1\right)-\lambda_{2}\left(\int \mathcal{D} X P_{X}[X]|X|^{2}-P\right)
$$

where $\lambda_{1,2}$ are Lagrange multipliers. We substitute $H[Y]$ and $H[Y \mid X]$ from Eqs. (39) and (40) to (41), perform the variation of the functional $J\left[P_{X}, \lambda_{1}, \lambda_{2}\right]$ over $P_{X}[X], \lambda_{1}, \lambda_{2}$, and write the Euler-Lagrange equations $\delta J\left[P_{X}, \lambda_{1}, \lambda_{2}\right]=0$ :

$$
\begin{aligned}
& \int \mathcal{D} X P_{X}[X]=1, \\
& \int \mathcal{D} X P_{X}[X]|X|^{2}=P, \\
& -1-\log P_{X}[X]-\frac{1}{2} \log \left(1+\frac{\gamma^{2} L^{2}}{3}|X|^{4}\right)- \\
& \lambda_{1}-\lambda_{2}|X|^{2}=0 .
\end{aligned}
$$

The solution $P_{X}^{o p t}[X]$ of Eqs. (42)-(44) referred to as the "optimal" distribution depends only on $|X|$ and has the form:

$$
P_{X}^{o p t}[X]=N_{0}(P) \frac{\exp \left\{-\lambda_{0}(P)|X|^{2}\right\}}{\sqrt{1+\gamma^{2} L^{2}|X|^{4} / 3}}
$$

where functions $N_{0}(P)$ and $\lambda_{0}(P)$ are determined from the conditions (42), (43):

$$
\int \mathcal{D} X P_{X}^{o p t}[X]=2 \pi N_{0}(P) \int_{0}^{\infty} \frac{d \rho \rho e^{-\lambda_{0}(P) \rho^{2}}}{\sqrt{1+\gamma^{2} L^{2} \rho^{4} / 3}}=1
$$




$$
\int \mathcal{D} X P_{X}^{o p t}[X]|X|^{2}=2 \pi N_{0}(P) \int_{0}^{\infty} \frac{d \rho \rho^{3} e^{-\lambda_{0}(P) \rho^{2}}}{\sqrt{1+\gamma^{2} L^{2} \rho^{4} / 3}}=P
$$

In a parametric form this dependance reads

$$
\lambda_{0}(P)=\frac{\gamma L}{\sqrt{3}} \alpha, \quad N_{0}(P)=\frac{\gamma L}{\pi \sqrt{3} G(\alpha)},
$$

here $G(\alpha)=\int_{0}^{\infty} d z e^{-\alpha z} / \sqrt{1+z^{2}}=\frac{\pi}{2}\left(H_{0}(\alpha)-Y_{0}(\alpha)\right)$ with $Y_{0}(\alpha)$ and $H_{0}(\alpha)$ being the Neumann and Struve functions of zero order, respectively. The parameter $\alpha(P)>0$ emerges as the real solution of the nonlinear equation $\frac{d}{d \alpha} \log G(\alpha)=-\gamma L P / \sqrt{3}$, which comes from Eqs. (46) and (47). Let us emphasize that the optimal distribution obtained here, $P_{X}^{o p t}[X](45)$, is different from the half-Gaussian distribution, see Eq. (33) for $\beta=1$, whereas in the Ref. [14] the half-Gaussian distribution was considered as optimal. For sufficiently large values of the power $P$, such that $\log (\gamma P L) \gg 1$, we can simplify (48) using the asymptotic expansions of $Y_{0}(\alpha)$ and $H_{0}(\alpha)$ at small $\alpha$, see [22]:

$$
\begin{aligned}
\lambda_{0}(P) & \approx \frac{1-\log \log (C \tilde{\gamma}) / \log (C \tilde{\gamma})}{P \log (C \tilde{\gamma})}, \\
N_{0}(P) & \approx \frac{\tilde{\gamma}}{\pi P} \log ^{-1}\left[C \tilde{\gamma} /\left(P \lambda_{0}(P)\right)\right],
\end{aligned}
$$

where $C=2 e^{-\gamma_{E}}$ and $\tilde{\gamma}=\gamma L P / \sqrt{3}$. At small $P$, the parameter $\tilde{\gamma} \ll 1$, the solution of the Eqs. (46) and (47) has the form:

$$
\lambda_{0}(P)=\frac{1}{P}\left(1-2 \tilde{\gamma}^{2}\right), N_{0}(P)=\frac{1}{\pi P}\left(1-\tilde{\gamma}^{2}\right) .(50
$$

It is worth noting that at $\tilde{\gamma} \rightarrow 0$ our distribution (45) approaches the Gaussian distribution (34) that is known to be optimal for the linear channel [1]. In Ref. [20] we found the first correction to $P_{X}^{o p t}[X]$ proportional to $Q L$. In Fig. 1 and Fig. 2 we demonstrate the behavior of the product $P \lambda_{0}(P)$ and $\pi P N_{0}(P)$, correspondingly, together with the asymptotics (49) and (50) as the functions of dimensionless parameter $\tilde{\gamma}$.

\section{THE MUTUAL INFORMATION}

Now we are ready to consider the mutual information for different distributions. We start our consideration from the mutual information for the optimal input signal distribution $P_{X}^{o p t}[X]$.

To calculate the mutual information we substitute the expression (45) for $P_{X}^{o p t}[X]$ in equations (39)-(40) and using the definition (4) we obtain

$$
I_{P_{X}^{o p t}[X]}=P \lambda_{0}(P)-\log N_{0}(P)-\log (\pi e Q L) .
$$

This equation gives the mutual information $I_{P_{X}^{o p t}[X]}$ up to terms proportional to $Q L$.

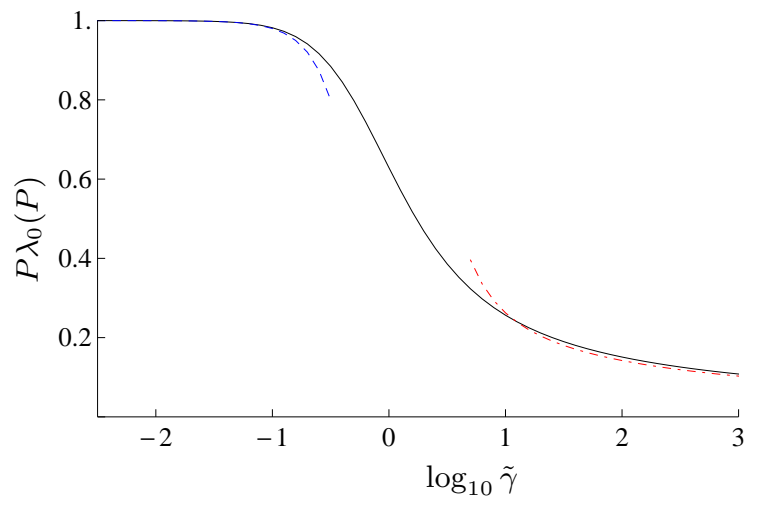

Figure 1: The product $P \lambda_{0}(P)$ as the function of dimensionless parameter $\tilde{\gamma}$ in the logarithmic scale. The solid black line corresponds to precise value of the product $P \lambda_{0}(P)$, see Eq. (48); the red dashed dotted line corresponds to the asymptotics (49) of $P \lambda_{0}(P)$; the blue dashed line corresponds to the asymptotics (50) of $P \lambda_{0}(P)$.

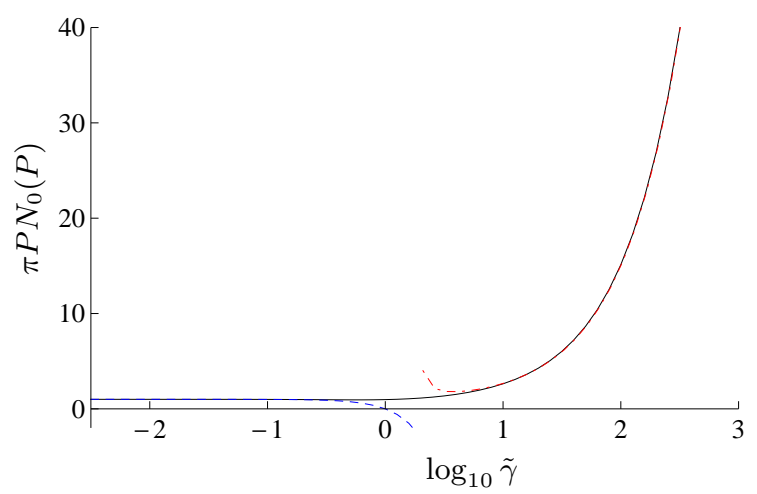

Figure 2: The product $\pi P N_{0}(P)$ as the function of dimensionless parameter $\tilde{\gamma}$ in the logarithmic scale. The solid black line corresponds to precise value of the product $\pi P N_{0}(P)$, see Eq. (48); the red dashed dotted line corresponds to the asymptotics (49) of $\pi P N_{0}(P)$; the blue dashed line corresponds to the asymptotics (50) of $\pi P N_{0}(P)$.

The mutual information (51) is depicted by the black solid line in Fig. 3 as a function of signal power $P$ for the following parameters: $Q=1.5 \times 10^{-7} \mathrm{~mW} \mathrm{~km}^{-1}$, $\gamma=10^{-3} \mathrm{~mW}^{-1} \mathrm{~km}^{-1}, L=1000 \mathrm{~km}$. For these realistic parameters the power range (23) is actually very wide:

$$
1.5 \times 10^{-4} \mathrm{~mW} \ll P \ll 0.66 \times 10^{4} \mathrm{~mW} .
$$

There is no simple analytical form for $N_{0}(P)$ and $\lambda_{0}(P)$ therefore to plot Fig. 3, Fig 4, and Fig 5 (see below) we calculated $\lambda_{0}(P)$ and $N_{0}(P)$ numerically. For large and small values of the parameter $\tilde{\gamma}$ we can use the solutions in Eqs. (49) and (50), respectively. At small $\tilde{\gamma}=\gamma L P / \sqrt{3}$ we obtain

$$
I_{P_{X}^{o p t}[X]} \approx \log (1+\mathrm{SNR})-\tilde{\gamma}^{2},
$$

which is simply the Shannon capacity $\log (1+\mathrm{SNR})$ at large SNR of the linear channel (1) with the first nonlinear correction. In Eq. (53) the unity in the logarithm 


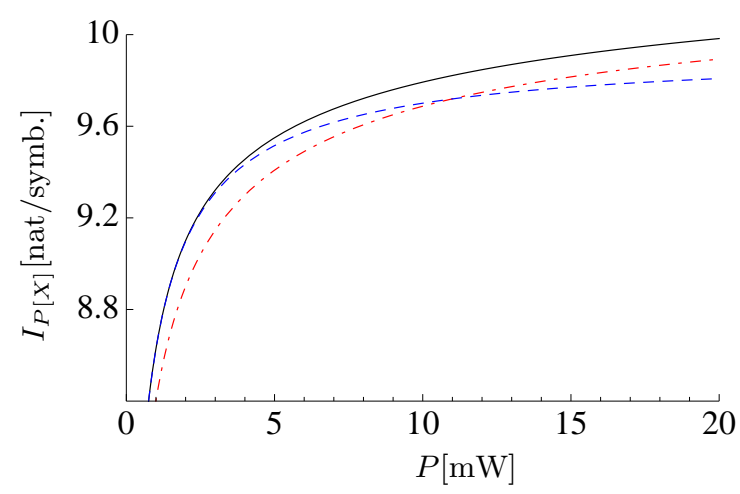

Figure 3: The mutual information for various input PDFs as a function of input average power $P$ for the parameters $Q=$ $1.5 \times 10^{-7} \mathrm{~mW} \mathrm{~km}^{-1}, \gamma=10^{-3} \mathrm{~mW}^{-1} \mathrm{~km}^{-1}, L=1000 \mathrm{~km}$. (a): The solid black line, blue dashed line, red dashed dotted line correspond to the optimal PDF $P_{X}^{o p t}[X]$, Gaussian PDF $P_{X}^{(2)}[X]$, and half-Gaussian $\operatorname{PDF} P_{X}^{(1)}[X]$, respectively.

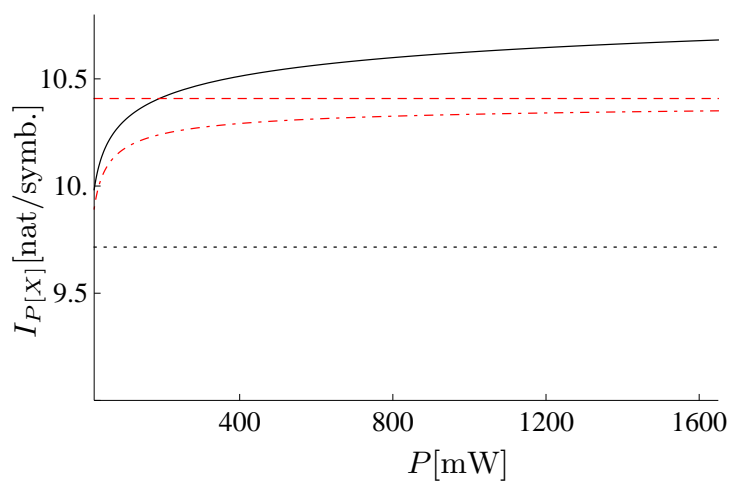

Figure 4: The mutual information for various input PDFs as a function of input average power $P$ for the parameters $Q=$ $1.5 \times 10^{-7} \mathrm{~mW} \mathrm{~km}^{-1}, \gamma=10^{-3} \mathrm{~mW}^{-1} \mathrm{~km}^{-1}, L=1000 \mathrm{~km}$. The solid black line corresponds to $I_{P_{X}^{o p t}[X]}$, see Eq. (51); the red dashed dotted line corresponds to the mutual information for the half-Gaussian distribution $I_{P_{X}^{(1)}[X]}$, see Eq. (57) for $\beta=1$; the red dashed line corresponds to our limit (59) at $\tilde{\gamma} \gg 1$ for the half-Gaussian distribution; the black dotted line corresponds to the result [14], see Eq. (10).

is beyond the accuracy of our calculation but we keep it to bring to notice that the derived expressions (40) and (53) have the correct limit when the parameter $\gamma$ tends to zero (in contrast to the Eq. (35) in Ref. [14]). In the power sub-interval $(\gamma L)^{-1} \ll P \ll\left(Q L^{3} \gamma^{2}\right)^{-1}$ using Eq. (49) one can obtain following expression for the mutual information

$$
\begin{aligned}
& I_{P_{X}^{o p t}[X]}=\log \log (C \gamma L P / \sqrt{3})-\log \left(Q L^{2} \gamma e / \sqrt{3}\right)+ \\
& \frac{1}{\log (C \gamma L P / \sqrt{3})}[\log \log (C \gamma L P / \sqrt{3})+1- \\
& \left.\frac{\log \log (C \gamma L P / \sqrt{3})}{\log (C \gamma L P / \sqrt{3})}\right]
\end{aligned}
$$

This equation is obtained with the accuracy $1 / \log ^{2}(\gamma L P)$. One can see that the mutual information $I_{P_{X}^{o p t}[X]}$ grows as $\log \log P$.

In the remainder of this Section we perform an analysis of the mutual information for the distribution $P_{X}^{(\beta)}[X]$, see Eq. (33), generalizing the half-Gaussian distribution (8) (see, for example Ref. [14]) and the Gaussian input PDF (34). In the leading order in $1 / \mathrm{SNR}$ from (39) we obtain

$$
H_{\beta}[Y]=\log \left(P \frac{2 \pi}{\beta} \Gamma\left(\frac{\beta}{2}\right)\right)+\frac{\beta}{2}+\frac{2-\beta}{2} \psi\left(\frac{\beta}{2}\right),
$$

where $\psi(z)$ is the digamma function $\psi(z)=\Gamma^{\prime}(z) / \Gamma(z)$, where $\psi(1)=-\gamma_{E}$ and $\psi(1 / 2)=-\gamma_{E}-2 \log (2)$. The substitution of Eq. (33) into Eq. (40) gives

$$
\begin{aligned}
H_{\beta}[Y \mid X]= & \int_{0}^{\infty} d \tau \frac{e^{-\tau} \tau^{\frac{\beta}{2}-1}}{2 \Gamma\left(\frac{\beta}{2}\right)} \log \left(1+\frac{4 \tilde{\gamma}^{2} \tau^{2}}{\beta^{2}}\right)+ \\
& \log (\pi e Q L) .
\end{aligned}
$$

The integral in Eq. (56) can be calculated analytically using Ref. [22], however, the result of the integration is cumbersome, hence we do not present it here. One can easily obtain the mutual information $I_{P_{X}^{(\beta)}[X]}$ by subtracting Eq. (56) from Eq. (55):

$$
\begin{aligned}
& I_{P_{X}^{(\beta)}}[X]=\log \mathrm{SNR}+\log \left(\frac{2 \Gamma(\beta / 2)}{\beta}\right)- \\
& \int_{0}^{\infty} d \tau \frac{e^{-\tau} \tau^{\frac{\beta}{2}-1}}{2 \Gamma\left(\frac{\beta}{2}\right)} \log \left(1+\frac{4 \tilde{\gamma}^{2} \tau^{2}}{\beta^{2}}\right)+ \\
& \frac{\beta-2}{2}\left(1-\psi\left(\frac{\beta}{2}\right)\right) .
\end{aligned}
$$

The mutual information is depicted in Fig. 3 for the Gaussian distribution by the blue dashed line, and for the half-Gaussian by the red dashed dotted line. One can see that at small $P$ the mutual information for the Gaussian distribution is greater than that of the halfGaussian, whereas at $P>11 \mathrm{~mW}$ the mutual information is greater for the half-Gaussian distribution. Note that $I_{P_{X}^{o p t}[X]}$ is greater than $I_{P_{X}^{(\beta)}[X]}$ for all values of $P$, as it should be. At $\tilde{\gamma} \gg 1$ the mutual information $I_{P_{X}^{(\beta)}[X]}$ takes the form

$$
\begin{aligned}
I_{P_{X}^{(\beta)}[X]}= & -\log \left(Q L^{2} \gamma\right)-\frac{2-\beta}{2}+\frac{\log 3}{2}- \\
& \frac{\beta}{2} \psi\left(\frac{\beta}{2}\right)+\log (\Gamma(\beta / 2)) .
\end{aligned}
$$

One can see that at large SNR $I_{P_{X}^{(\beta)}[X]}$ goes to a constant in the interval of power $P$ considered, and this constant depends on the noise power $Q L$. We remind that $I_{P_{X}^{o p t}[X]}$ 


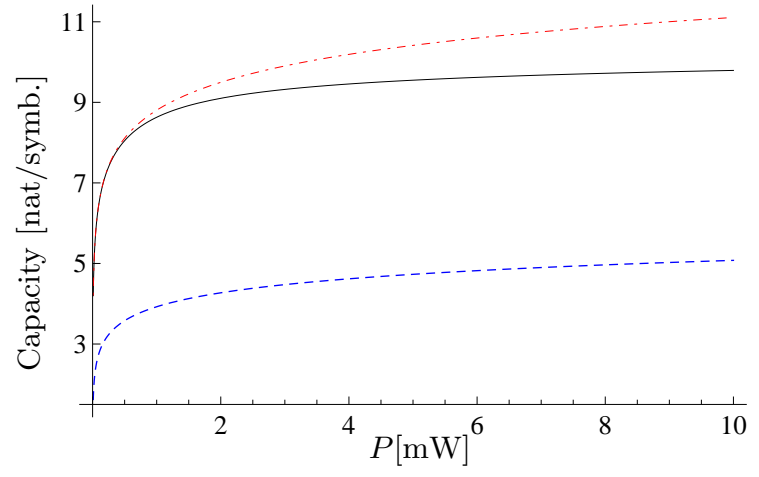

Figure 5: Shannon capacity, the capacity of the nonlinear channel $I_{P_{X}^{o p t}[X]}$, and the asymptotic capacity bound (9) from Ref. [13] for the parameters $Q=1.5 \times 10^{-7} \mathrm{~mW} \mathrm{~km}^{-1}, \gamma=$ $10^{-3} \mathrm{~mW}^{-1} \mathrm{~km}^{-1}, L=1000 \mathrm{~km}$. The red dashed-dotted line corresponds to the Shannon limit $\log [1+\mathrm{SNR}]$, the black solid line corresponds to $I_{P_{X}^{o p t}[X]}$, see Eq. (51), the blue dashed line corresponds to the bound (9).

increases as $\log \log P$ in the interval under consideration. The mutual information for the half-Gaussian distribution (8) in the regime $\tilde{\gamma} \gg 1$ can be obtained as a particular case of (58) for $\beta=1$ :

$$
\begin{aligned}
I_{P_{X}^{(1)}[X]}= & -\log \left(Q L^{2} \gamma\right)+\frac{\log 3 \pi-1+\gamma_{E}}{2}+ \\
& \log 2 .
\end{aligned}
$$

Comparing our expression (59) with the result (40) of Ref. [14] we have an extra term $+\log 2$ due to our more accurate calculation of $H[Y \mid X]$. Our result (59) and the result of Ref. [14], see Eq. (10), are presented in Fig. 4. In Fig. 4 one can see that the mutual information (51) for the optimal distribution exceeds the limit (59) at $P \sim$ $190 \mathrm{~mW}$. At this power the difference between the limit (59) and $I_{P_{X}^{(1)}[X]}$ evaluated on the base of Eq. (57) with $\beta=1$ is of order of $1.5 \%$ and getting smaller at higher $P$. Also the capacity bound from Ref. [14], see Eq. (10) therein, is plotted by the black dotted line in Fig. 4 .

Since we have now found $P_{X}^{o p t}[X]$ in the power range (23), we can calculate an approximation for the capacity of the considered per-sample nonlinear channel. By definition it coincides with the mutual information expression (51):

$$
C=I_{P_{X}^{o p t}[X]} \cdot
$$

Let us emphasize that this result for the capacity is valid up to terms proportional to $Q L$. The correction to the capacity proportional to $Q L$ can be found in Ref. [20].

The comparison of the approximation (60) with the Shannon capacity of the linear channel with Gaussian noise and with the asymptotic capacity bound (9) from Ref. [13] is presented in Fig. 5. One can see that the Shannon capacity of the linear channel with Gaussian noise is always greater than the approximation (60) for the nondispersive nonlinear fiber channel for the considered range of $P$. But the approximation (60) is greater than the asymptotic capacity bound (9) in the intermediate power range $(23)$.

\section{CONCLUSION}

We have developed a new approach to the calculation of the conditional PDF via the path-integral representation (14) at large signal-to-noise ratio for the intermediate power range (23). This approach may be an especially useful technique for complex nonlinear channels in which the calculation of the conditional PDF is technically challenging. Applying our method to the persample nondispersive nonlinear fiber channel, we derived the compact analytical expressions for the conditional PDF, conditional entropy and the entropy of the output signal for different input signal PDFs $P_{X}[X]$. Moreover, we solved the variational problem on $P_{X}[X]$ maximizing the mutual information in the leading order in the noise power $Q L$ in the power range (23). It allows us to find the optimal input signal distribution (45) and the approximation for the channel capacity (51) in the power interval $Q L \ll P \ll\left(\gamma^{2} Q L^{3}\right)^{-1}$, which is extremely wide for realistic parameters, see (52). The found distribution $P_{X}^{o p t}[X]$ is different from the half-Gaussian one, and at the zero nonlinearity $P_{X}^{o p t}[X]$ approaches the Gaussian distribution. We demonstrated that the capacity in the power sub-interval $(\gamma L)^{-1} \ll P \ll\left(Q L^{3} \gamma^{2}\right)^{-1}$ grows as $\log \log P$ rather than has constant behavior obtained in Ref. [14]. In that sub-interval the found capacity is greater than the bound (9) obtained in Ref. [13] and lower than the Shannon capacity of the linear channel with the Gaussian noise.

\section{Acknowledgments}

The calculations of the next-to-leading order corrections have been performed with support of the the Russian Science Foundation (RSF) (Grant No. 16-1110133). Part of the work (Section III) was supported by the Russian Scientific Foundation (RFBR), Grant No. 16-31-60031. A. V. R. thanks the President program (SP-2415.2015.2) for support. The work of S.K.T. was supported by the grant of the Ministry of Education and Science of the Russian Federation (Agreement No. 14.B25.31.0003) and the EPSRC project UNLOC.

\section{Appendix A: THE CLASSICAL SOLUTION $\Psi_{c l}$ AND THE ACTION $S\left[\Psi_{c l}\right]$.}

In Ref. [17] we have shown that in the case $\mathrm{SNR}=$ $P / Q L \gg 1$ the conditional probability can be written in 
the form:

$$
\begin{aligned}
& P[Y \mid X]=\exp \left\{-\frac{S\left[\Psi_{c l}(z)\right]}{Q}\right\} \times \\
& \psi(L)=0 \\
& \int_{\psi(0)=0} \mathcal{D} \psi \exp \left\{-\frac{S\left[\Psi_{c l}(z)+\psi(z)\right]-S\left[\Psi_{c l}(z)\right]}{Q}\right\}
\end{aligned}
$$

where for the nondispersive model the effective action reads

$$
S[\psi]=\left.\left.\int_{0}^{L} d z\left|\partial_{z} \psi-i \gamma\right| \psi\right|^{2} \psi\right|^{2} .
$$

The action (A2) is associated with the l.h.s. of the nonlinear Shrödinger equation

$$
\partial_{z} \psi(z)-i \gamma|\psi(z)|^{2} \psi(z)=\eta(z),
$$

where the noise $\eta(z)$ has the Gaussian nature:

$$
\langle\eta(z)\rangle_{\eta}=0, \quad\left\langle\eta(z) \bar{\eta}\left(z^{\prime}\right)\right\rangle_{\eta}=Q \delta\left(z-z^{\prime}\right) .
$$

The measure $\mathcal{D} \psi$ in Eq. (A1) is defined as

$$
\mathcal{D} \psi=\lim _{\Delta \rightarrow 0}\left(\frac{1}{\Delta \pi Q}\right)^{N} \prod_{i=1}^{N-1} d \operatorname{Re} \psi_{i} d \operatorname{Im} \psi_{i}
$$

here $\psi_{i}=\psi\left(z_{i}\right)$ and $\Delta=\frac{L}{N}$ is the grid space.

Now we consider the difference of actions in the exponent of the path-integral in Eq. (A1).

$$
\begin{aligned}
& S\left[\Psi_{c l}(z)+\psi(z)\right]-S\left[\Psi_{c l}(z)\right]= \\
& \int_{0}^{L} d z\left\{\left|\partial_{z} \psi-i \gamma\left(2 \psi\left|\Psi_{c l}\right|^{2}+\bar{\psi} \Psi_{c l}^{2}\right)\right|^{2}+\right. \\
& 2 \gamma \operatorname{Im}\left[\left(\partial_{z} \bar{\Psi}_{c l}+i \gamma \bar{\Psi}_{c l}\left|\Psi_{c l}\right|^{2}\right)\left(2 \Psi_{c l}|\psi|^{2}+\bar{\Psi}_{c l} \psi^{2}\right)\right]+ \\
& \left.\gamma^{2}\left|2 \Psi_{c l}\right| \psi\right|^{2}+\bar{\Psi}_{c l} \psi^{2}+\left.\psi|\psi|^{2}\right|^{2}+ \\
& 2 \gamma \operatorname{Im}\left[\left(\partial_{z} \bar{\psi}+i \gamma\left(2 \bar{\psi}\left|\Psi_{c l}\right|^{2}+\psi \bar{\Psi}_{c l}^{2}\right)\right) \times\right. \\
& \left.\left(2 \Psi_{c l}|\psi|^{2}+\bar{\Psi}_{c l} \psi^{2}+\psi|\psi|^{2}\right)\right]+ \\
& \left.2 \gamma \operatorname{Im}\left[\left(\partial_{z} \bar{\Psi}_{c l}+i \gamma \bar{\Psi}_{c l}\left|\Psi_{c l}\right|^{2}\right) \psi|\psi|^{2}\right]\right\} .
\end{aligned}
$$

In Eq. (A1) the function $\Psi_{c l}(z)$ is the solution of the equation $\delta S\left[\Psi_{c l}\right]=0$ (Euler-Lagrange equation) which has the form

$$
\frac{d^{2} \Psi_{c l}}{d z^{2}}-4 i \gamma\left|\Psi_{c l}\right|^{2} \frac{d \Psi_{c l}}{d z}-3 \gamma^{2}\left|\Psi_{c l}\right|^{4} \Psi_{c l}=0
$$

with boundary conditions $\Psi_{c l}(0)=X=|X| \exp \left[i \phi^{(X)}\right]$, $\Psi_{c l}(L)=Y=|Y| \exp \left[i \phi^{(Y)}\right]$. It is easy to find the solution of Eq. (A7) in the polar coordinate system: $\Psi_{c l}(z)=\rho(\zeta) e^{i \theta(\zeta)}, \zeta=z / L$. The solution depends on four real integration constants. We denote them as $E, \tilde{\mu}$, $\zeta_{0}$ and $\theta_{0}$. There are two different regimes of the solution: in the trigonometric regime one has $E=\frac{k^{2}}{2} \geq 0$, and in the hyperbolic regime $E=-\frac{k^{2}}{2} \leq 0$. For both cases instead of $E$ we introduce the non-negative parameter $k=\sqrt{2|E|}$.

In the trigonometric case $\left(E=\frac{k^{2}}{2} \geq 0\right)$ we have the solution for $\tilde{\mu} \geq k \geq 0$ :

$$
\begin{aligned}
& \rho^{2}(\zeta)=\frac{1}{2 L \gamma}\left(\tilde{\mu}+\sqrt{\tilde{\mu}^{2}-k^{2}} \cos \left[2 k\left(\zeta-\zeta_{0}\right)\right]\right), \\
& \theta(\zeta)=\frac{\tilde{\mu}}{2}\left(\zeta-\zeta_{0}\right)+\sqrt{\tilde{\mu}^{2}-k^{2}} \frac{\sin \left[2 k\left(\zeta-\zeta_{0}\right)\right]}{4 k}+ \\
& \arctan \left[\left(\tilde{\mu}-\sqrt{\tilde{\mu}^{2}-k^{2}}\right) \frac{\tan \left[k\left(\zeta-\zeta_{0}\right)\right]}{k}\right]+\theta_{0} .
\end{aligned}
$$

Here the integration constants $\tilde{\mu}, k$ and $\zeta_{0}$ must be found from the boundary conditions:

$$
\begin{aligned}
& |X|^{2}=\rho^{2}(0)=\frac{\tilde{\mu}+\sqrt{\tilde{\mu}^{2}-k^{2}} \cos \left[2 k \zeta_{0}\right]}{2 L \gamma}, \\
& |Y|^{2}=\rho^{2}(1)=\frac{\tilde{\mu}+\sqrt{\tilde{\mu}^{2}-k^{2}} \cos \left[2 k\left(1-\zeta_{0}\right)\right]}{2 L \gamma}, \quad(\mathrm{A} 10) \\
& \phi^{(X)}=\theta(0)=-\frac{\tilde{\mu}}{2} \zeta_{0}-\sqrt{\tilde{\mu}^{2}-k^{2}} \frac{\sin \left[2 k \zeta_{0}\right]}{4 k}- \\
& \arctan \left[\left(\tilde{\mu}-\sqrt{\tilde{\mu}^{2}-k^{2}}\right) \frac{\tan \left[k \zeta_{0}\right]}{k}\right]+\theta_{0}, \quad(\mathrm{~A} 11) \\
& \phi^{(Y)}=\theta(1)=\frac{\tilde{\mu}}{2}\left(1-\zeta_{0}\right)+\sqrt{\tilde{\mu}^{2}-k^{2}} \frac{\sin \left[2 k\left(1-\zeta_{0}\right)\right]}{4 k}+ \\
& \arctan \left[\left(\tilde{\mu}-\sqrt{\tilde{\mu}^{2}-k^{2}}\right) \frac{\tan \left[k\left(1-\zeta_{0}\right)\right]}{k}\right]+\theta_{0} .
\end{aligned}
$$

Then one can find the action

$$
\begin{aligned}
& S\left[\Psi_{c l}\left(z ; E=\frac{k^{2}}{2}, \tilde{\mu}, \zeta_{0}, \theta_{0}\right)\right]=\frac{k^{2}}{2 \gamma L}(\tilde{\mu}- \\
& \left.\sqrt{\tilde{\mu}^{2}-k^{2}} \frac{\sin \left[2 k\left(1-\zeta_{0}\right)\right]+\sin \left[2 k \zeta_{0}\right]}{2 k}\right) .
\end{aligned}
$$

In the hyperbolic case $\left(E=-\frac{k^{2}}{2} \leq 0\right)$ we have the solution for $k \geq 0$ and arbitrary $\tilde{\mu}$ in the following form

$$
\begin{aligned}
& \rho^{2}(\zeta)=\frac{-\tilde{\mu}+\sqrt{\tilde{\mu}^{2}+k^{2}} \cosh \left[2 k\left(\zeta-\zeta_{0}\right)\right]}{2 L \gamma}, \\
& \theta(\zeta)=-\frac{\tilde{\mu}}{2}\left(\zeta-\zeta_{0}\right)+\sqrt{\tilde{\mu}^{2}+k^{2}} \frac{\sinh \left[2 k\left(\zeta-\zeta_{0}\right)\right]}{4 k}- \\
& \arctan \left[\left(\tilde{\mu}+\sqrt{\tilde{\mu}^{2}+k^{2}}\right) \frac{\tanh \left[k\left(\zeta-\zeta_{0}\right)\right]}{k}\right]+\theta_{0},(\mathrm{~A} 14)
\end{aligned}
$$

where $\tilde{\mu}, k, \zeta_{0}$, and $\theta_{0}$ are derived from the same procedure as in the trigonometric regime. The action reads

$$
\begin{aligned}
& S\left[\Psi_{c l}\left(z ; E=-\frac{k^{2}}{2}, \tilde{\mu}, \zeta_{0}, \theta_{0}\right)\right]=\frac{k^{2}}{2 \gamma L}(\tilde{\mu}+ \\
& \left.\sqrt{\tilde{\mu}^{2}+k^{2}} \frac{\sinh \left[2 k\left(1-\zeta_{0}\right)\right]+\sinh \left[2 k \zeta_{0}\right]}{2 k}\right) .
\end{aligned}
$$


Note, there are two solutions of Eq. (A7) with constant $\rho(z)=\rho(0) \equiv \rho$ obeying only the input boundary condition $\Psi_{0}(0)=X$. The first one reads

$$
\Psi_{0}(z)=\rho \exp \left\{i \mu \frac{z}{L}+i \phi^{(X)}\right\}
$$

where $\mu=\gamma L \rho^{2}=\gamma L|X|^{2}$. This function corresponds to the solution representation (A8) with $k=0$ and $\tilde{\mu}=\mu$ or to the solution representation (A14) with $k=0$ and $\tilde{\mu}=-\mu$. The function $\Psi_{0}(z)$ is the solution of the Eq. (A3) with zero noise and with the input boundary condition. Furthermore, $\Psi_{0}(z)$ delivers the absolute minimum of the action $(\mathrm{A} 2): S\left[\Psi_{0}(z)\right]=0$. The second solution of Eq. (A7) with constant $\rho(z)$ is the trigonometric regime (A8) case with $\tilde{\mu}=k=2 \mu$ :

$$
\begin{aligned}
& \Psi_{\rho=\text { const }}(z)=\rho \exp \left\{3 i \mu \frac{z}{L}+i \phi^{(X)}\right\}, \\
& \mu=\gamma L \rho^{2}=\gamma L|X|^{2} .
\end{aligned}
$$

To find the solution of Eq. (A7) one should express the integration constant through the boundary conditions. Instead, we exploit the fact that the noise power $Q L$ is much less than the input signal power. In other words, we will find a solution of Eq. (A7) that is close to $\Psi_{0}(z)$ : it is the solution of Eq. (A3) with zero noise which provides the absolute minimum of the action $S\left[\Psi_{0}(z)\right]=0$. In that fashion we perform the substitution in Eq. (A7):

$$
\Psi_{c l}(z)=(\rho+\varkappa(z)) \exp \left\{i \mu \frac{z}{L}+i \phi^{(X)}\right\}
$$

where the function $\varkappa(z)$ is assumed to be small: $\varkappa(z) \ll \rho$ for all configurations of $\Psi_{c l}(z)$ providing $S\left[\Psi_{c l}(z)\right] / Q=$ $\mathcal{O}(1)$ when $Q L$ tends to zero. We have the following equation on $\varkappa(z)$ resulting from the Eq. (A7):

$$
\begin{aligned}
& \frac{d^{2} \varkappa}{d z^{2}}-2 i \frac{\mu}{L} \frac{d \varkappa}{d z}-4 \frac{\mu^{2}}{L^{2}} \operatorname{Re}[\varkappa]=4 i \frac{\mu}{L \rho}(\varkappa+\bar{\varkappa}) \frac{d \varkappa}{d z}+\frac{\mu^{2}}{L^{2} \rho}\left[5 \varkappa^{2}+10|\varkappa|^{2}+3 \bar{\varkappa}^{2}\right]+ \\
& \frac{|\varkappa|^{2} \mu}{L^{2} \rho^{2}}\left[4 i L \frac{d \varkappa}{d z}+9 \mu \bar{\varkappa}+14 \mu \varkappa\right]+\frac{3 \mu^{2}}{L^{2} \rho^{2}} \varkappa^{3}+\frac{3 \mu^{2}}{L^{2} \rho^{3}}|\varkappa|^{2}\left[3|\varkappa|^{2}+2 \varkappa^{2}\right]+\frac{3 \mu^{2}}{L^{2} \rho^{4}}|\varkappa|^{4} \varkappa .
\end{aligned}
$$

We present $\varkappa(z)$ as a perturbation theory decomposition in powers of $1 / \sqrt{\mathrm{SNR}}: \varkappa(z)=\varkappa_{1}(z)+\varkappa_{2}(z)+\ldots$, where $\varkappa_{1}(z)$ is of $1 / \sqrt{\mathrm{SNR}}$ order and provides the leading order contribution, $\varkappa_{2}(z)$ is of $1 / \mathrm{SNR}$ order, and so on.

The linearized equation for the function $\varkappa_{1}(z)=$ $x_{1}(z)+i y_{1}(z)$ can be obtained from Eq. (A19) by omitting the r.h.s. of this equation:

$$
\frac{d^{2} \varkappa_{1}}{d z^{2}}-2 i \frac{\mu}{L} \frac{d \varkappa_{1}}{d z}-4 \frac{\mu^{2}}{L^{2}} \operatorname{Re}\left[\varkappa_{1}\right]=0
$$

The boundary conditions $\Psi_{c l}(0)=X$ and $\Psi_{c l}(1)=Y \equiv$ $\rho^{\prime} e^{i \phi^{(Y)}}$ lead to

$$
\begin{aligned}
& \varkappa_{1}(0)=0, \\
& \varkappa_{1}(L)=x_{0}+i y_{0}=\rho^{\prime} e^{i\left(\phi^{(Y)}-\phi^{(X)}-\mu\right)}-\rho .
\end{aligned}
$$

The solution $\varkappa_{1}(z)=x_{1}(z)+i y_{1}(z)$ of the linearized boundary problem (A20), (A21) is polynomial

$$
\begin{aligned}
& x_{1}(z)=\left(-\mu a_{1}(X, Y) \frac{z}{L}+a_{2}(X, Y)\right) \frac{z}{L}, \\
& y_{1}(z)=\left(-\frac{2}{3} \mu^{2} a_{1}(X, Y) \frac{z^{2}}{L^{2}}+\right. \\
& \left.\mu a_{2}(X, Y) \frac{z}{L}+a_{1}(X, Y)\right) \frac{z}{L},
\end{aligned}
$$

where coefficients $a_{1}(X, Y)$ and $a_{2}(X, Y)$ can be found from the boundary conditions (A21) and have the form:

$$
\begin{aligned}
& a_{1}(X, Y)=\frac{-\mu x_{0}+y_{0}}{1+\mu^{2} / 3} \\
& a_{2}(X, Y)=\frac{\left(1-2 \mu^{2} / 3\right) x_{0}+\mu y_{0}}{1+\mu^{2} / 3}
\end{aligned}
$$

with $x_{0}=x_{0}(X, Y)$ and $y_{0}=y_{0}(X, Y)$ being determined from Eq. (A21). In the leading in $\sqrt{Q L}$ order the action reads

$$
\begin{aligned}
& \frac{1}{Q} S\left[\Psi_{0}(z)+\varkappa_{1}(z) e^{i \mu \frac{z}{L}+i \phi^{(X)}}\right] \approx \\
& \frac{1}{Q} \int_{0}^{L} d z\left[\left|\partial_{z} \varkappa_{1}-2 i \frac{\mu}{L} \operatorname{Re}\left[\varkappa_{1}\right]\right|^{2}\right]= \\
& =\frac{\left(1+4 \mu^{2} / 3\right) a_{1}^{2}-2 \mu a_{1} a_{2}+a_{2}^{2}}{Q L}= \\
& \frac{\left(1+4 \mu^{2} / 3\right) x_{0}^{2}-2 \mu x_{0} y_{0}+y_{0}^{2}}{Q L\left(1+\mu^{2} / 3\right)} .
\end{aligned}
$$

Let us proceed to the next-to-leading order corrections to $P[Y \mid X]$. We should calculate the next approximation $\varkappa_{2}(z)$ to the solution (A18). Taking into account 
Eq. (A20) we present the equation for $\varkappa_{2}(z)$ in the form

$$
\begin{aligned}
& \frac{d^{2} \varkappa_{2}}{d z^{2}}-2 i \frac{\mu}{L} \frac{d \varkappa_{2}}{d z}-4 \frac{\mu^{2}}{L^{2}} \operatorname{Re}\left[\varkappa_{2}\right]= \\
& 4 i \frac{\mu}{L \rho}\left(\varkappa_{1}+\bar{\varkappa}_{1}\right) \frac{d \varkappa_{1}}{d z}+ \\
& \frac{\mu^{2}}{L^{2} \rho}\left[5 \varkappa_{1}^{2}+10\left|\varkappa_{1}\right|^{2}+3 \bar{\varkappa}_{1}^{2}\right],
\end{aligned}
$$

where the boundary conditions for $\varkappa_{2}(z) \operatorname{read} \varkappa_{2}(0)=$ $\varkappa_{2}(L)=0$. The solution $\varkappa_{2}(z)=x_{2}(z)+i y_{2}(z)$ of Eq. (A25) is polynomial in $z$ and quadratic in $x_{0}$ and $y_{0}$ :

$$
\begin{aligned}
& x_{2}(z)=-\frac{\mu / \rho}{270\left(1+\mu^{2} / 3\right)^{3}}\left(1-\frac{z}{L}\right) \frac{z}{L} \times \\
&\left\{\mu\left(2 \mu^{4}-15 \mu^{2}+585\right) x_{0}^{2}+2\left(13 \mu^{2}\left(\mu^{2}+15\right)-180\right) x_{0} y_{0}+\mu\left(2 \mu^{2}+15\right)\left(5 \mu^{2}-9\right) y_{0}^{2}-\right. \\
& 5\left(\mu^{2}+3\right) \frac{z}{L}\left(\mu\left(\mu^{2}-15\right) x_{0}^{2}-4\left(\mu^{2}-6\right) x_{0} y_{0}+\mu\left(\mu^{2}+9\right) y_{0}^{2}\right)+ \\
& 5 \mu\left(\mu^{2}+3\right) \frac{z^{2}}{L^{2}}\left(3\left(5 \mu^{2}-3\right) x_{0}^{2}-36 \mu x_{0} y_{0}-\left(\mu^{2}-15\right) y_{0}^{2}\right)+ \\
&\left.20 \mu^{2}\left(\mu^{2}+3\right) \frac{z^{3}}{L^{3}}\left(y_{0}-\mu x_{0}\right)\left(2 \mu y_{0}-\left(\mu^{2}-3\right) x_{0}\right)-20 \mu^{3}\left(\mu^{2}+3\right) \frac{z^{4}}{L^{4}}\left(y_{0}-\mu x_{0}\right)^{2}\right\} . \\
& y_{2}(z)=- \frac{\mu / \rho}{270\left(1+\mu^{2} / 3\right)^{3}}\left(1-\frac{z}{L}\right) \frac{z}{L} \times \\
&\{\left(7 \mu^{4}-75 \mu^{2}+360\right) x_{0}^{2}+6 \mu\left(\mu^{2}+75\right) x_{0} y_{0}+3 \mu^{2}\left(5 \mu^{2}+39\right) y_{0}^{2}+ \\
& 2 \frac{z}{L}\left(\left(\mu^{6}-4 \mu^{4}+255 \mu^{2}+180\right) x_{0}^{2}+\mu\left(\mu^{2}+15\right)\left(13 \mu^{2}+3\right) x_{0} y_{0}+\mu^{2}\left(5 \mu^{4}+36 \mu^{2}-9\right) y_{0}^{2}\right)- \\
&\left.14 \mu\left(\mu^{2}+3\right) \frac{z^{2}}{L^{2}}\left(y_{0}-\mu x_{0}\right)\left(\left(15-4 \mu^{2}\right) x_{0}+9 \mu y_{0}\right)+84 \mu^{2}\left(\mu^{2}+3\right) \frac{z^{3}}{L^{3}}\left(y_{0}-\mu x_{0}\right)^{2}\right\} .
\end{aligned}
$$

In the leading, see Eq. (A24), and next-to-leading order in $\sqrt{Q L}$ the action reads

$$
\begin{aligned}
& \frac{1}{Q} S\left[\Psi_{c l}(z)\right] \approx \frac{\left(1+4 \mu^{2} / 3\right) x_{0}^{2}-2 \mu x_{0} y_{0}+y_{0}^{2}}{Q L\left(1+\mu^{2} / 3\right)}+\frac{\mu / \rho}{135 Q L\left(1+\mu^{2} / 3\right)^{3}}\left\{\mu\left(4 \mu^{4}+15 \mu^{2}+225\right) x_{0}^{3}+\right. \\
& \left.\left(23 \mu^{4}+255 \mu^{2}-90\right) x_{0}^{2} y_{0}+\mu\left(20 \mu^{4}+117 \mu^{2}-45\right) x_{0} y_{0}^{2}-3\left(5 \mu^{4}+33 \mu^{2}+30\right) y_{0}^{3}\right\}
\end{aligned}
$$

\section{Appendix B: THE PATH-INTEGRAL CALCULATION.}

To calculate the conditional probability density $P[Y \mid X]$ in Eq. (A1) one should find the pre-exponent path-integral, referred to as the quantum corrections near the classical solution $\Psi_{c l}(z)$, in the leading and next-to-

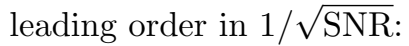

$$
I_{Q C}\left[\Psi_{c l}(z)\right]=\int_{\psi(0)=0}^{\psi(L)=0} \mathcal{D} \psi e^{-\frac{S\left[\Psi_{c l}(z)+\psi(z)\right]-S\left[\Psi_{c l}(z)\right]}{Q}} .
$$

In what follows we are interested in the leading and nextto-leading order corrections for the path-integral (A1).
That is why we retain only quadratic in $\psi$ terms in Eq. (A6). All these terms are placed in the second line of Eq. (A6). As it will be demonstrated below an extra power of $\psi$ results in an extra power of $\sqrt{Q L}$. In the leading and next-to-leading order calculation of the pathintegral we should take into account the first correction $\left(\varkappa_{1}(z) \propto \sqrt{Q L}\right)$ to the solution $\Psi_{c l}(z)$, see Eqs. (A18) and (A22). Now we put (A18) with $\varkappa_{1}(z)$ and $\psi(z)$ in the form $\psi(z)=u(z) \exp \left\{i \mu \frac{z}{L}+i \phi^{(X)}\right\}$ into the second and third lines of Eq. (A6). In our approximation we obtain 


$$
\begin{aligned}
& S\left[\Psi_{c l}(z)+\psi(z)\right]-S\left[\Psi_{c l}(z)\right] \approx \int_{0}^{L} d z\left\{\left|\partial_{z} u-i \frac{\mu}{L}(u+\bar{u})\right|^{2}+\right. \\
& \left.2 \frac{\mu}{L \rho} \operatorname{Im}\left[2\left(\partial_{z} \bar{u}+i \frac{\mu}{L}(u+\bar{u})\right)\left(u\left(\varkappa_{1}+\bar{\varkappa}_{1}\right)+\bar{u} \varkappa_{1}\right)+\left(\partial_{z} \bar{\varkappa}_{1}+i \frac{\mu}{L}\left(\varkappa_{1}+\bar{\varkappa}_{1}\right)\right)\left(2|u|^{2}+u^{2}\right)\right]\right\} .
\end{aligned}
$$

We substitute this difference in the exponent in Eq. (B1). Then we expand the exponent at small $Q$ and obtain the following expression within the accuracy of terms proportional to $Q L$ :

$$
\begin{aligned}
& \exp \left\{-\frac{S\left[\Psi_{c l}(z)+\psi(z)\right]-S\left[\Psi_{c l}(z)\right]}{Q}\right\} \approx \exp \left\{-\frac{1}{Q} \int_{0}^{L} d z\left|\partial_{z} u-i \frac{\mu}{L}(u+\bar{u})\right|^{2}\right\}\{1- \\
& \left.\frac{2 \mu}{Q L \rho} \operatorname{Im} \int_{0}^{L} d z\left[2\left(\partial_{z} \bar{u}+i \frac{\mu}{L}(u+\bar{u})\right)\left(u\left(\varkappa_{1}+\bar{\varkappa}_{1}\right)+\bar{u} \varkappa_{1}\right)+\left(\partial_{z} \bar{\varkappa}_{1}+i \frac{\mu}{L}\left(\varkappa_{1}+\bar{\varkappa}_{1}\right)\right)\left(2|u|^{2}+u^{2}\right)\right]\right\} .
\end{aligned}
$$

Here we imply that any extra power of $u$ or $\varkappa$ is suppressed by the multiplicative parameter $\sqrt{Q L}$, because at small $Q$ the main contribution to the path-integral comes from $u \sim \sqrt{Q L}$. We substitute this expansion (B3) into the path-integral (B1) and change the variable from $\psi(z)$ to $u(z)$ and arrive at

$$
\begin{aligned}
& I_{Q C}\left[\Psi_{0}(z)\right]=\int_{u(0)=0}^{u(L)=0} \mathcal{D} u e^{-\frac{\int_{0}^{L} d z\left|\partial_{z} u-i \frac{\mu}{L}(u+\bar{u})\right|^{2}}{Q}} \times \\
& {\left[1-\frac{4 \mu}{Q L \rho} \operatorname{Im} \int_{0}^{L} d z\left\{\left(\partial_{z} \bar{\varkappa}_{1}+i \frac{\mu}{L}\left(\varkappa_{1}+\bar{\varkappa}_{1}\right)\right)\left(|u|^{2}+\frac{u^{2}}{2}\right)+\right.\right.} \\
& \left.\left.\left(\partial_{z} \bar{u}+i \frac{\mu}{L}(u+\bar{u})\right)\left(u\left(\varkappa_{1}+\bar{\varkappa}_{1}\right)+\bar{u} \varkappa_{1}\right)\right\}\right] .
\end{aligned}
$$

To calculate the leading and next-to-leading order contributions to $I_{Q C}\left[\Psi_{0}(z)\right]$ in $\sqrt{Q L}$ we should take the first and the second terms in the square brackets in Eq. (B4), respectively. We start our consideration from the leading order. In this case we represent the path-integral

$$
I_{Q C}^{(0)}\left[\Psi_{0}(z)\right]=\int_{u(0)=0}^{u(L)=0} \mathcal{D} u e^{-\frac{\int_{0}^{L} d z\left|\partial_{z} u-i \frac{\mu}{L}(u+\bar{u})\right|^{2}}{Q}}
$$

in the retarded discretization scheme:

$$
\begin{aligned}
& I_{Q C}^{(0)}\left[\Psi_{0}(z)\right]=\lim _{N \rightarrow \infty}\left(\frac{N}{\pi Q L}\right)^{N} \times \\
& \int_{-\infty}^{\infty} \prod_{i=1}^{N-1} d u_{i}^{(1)} d u_{i}^{(2)} \exp \left\{-\frac{N}{Q L} \sum_{i=0}^{N-1}\left[\left(u_{i+1}^{(1)}-u_{i}^{(1)}\right)^{2}+\right.\right. \\
& \left.\left.\left(u_{i+1}^{(2)}-u_{i}^{(2)}-2 \frac{\mu}{N} u_{i}^{(1)}\right)^{2}\right]\right\}
\end{aligned}
$$

where we use the measure (A5) and the notations $u\left(z_{j}\right)=$ $u_{j}^{(1)}+i u_{j}^{(2)}, z_{i}=\Delta i, \Delta=\frac{L}{N}$ and $u_{0}^{(1)}=u_{N+1}^{(1)}=u_{0}^{(2)}=$ $u_{N+1}^{(2)}=0$. The sequential integration over $u_{N-1}^{(2)}, u_{N-2}^{(2)}$, $\ldots, u_{1}^{(2)}$ is trivial:

$$
\begin{aligned}
& \int d Y \exp \left\{-\frac{(A-Y)^{2}}{2 \tau_{1}}-\frac{(Y-B)^{2}}{2 \tau_{2}}\right\}= \\
& \left(2 \pi \frac{\tau_{1} \tau_{2}}{\tau_{1}+\tau_{2}}\right)^{1 / 2} \exp \left\{-\frac{(A-B)^{2}}{2\left(\tau_{1}+\tau_{2}\right)}\right\} .
\end{aligned}
$$

It leads to the remaining integral (over $u_{i}^{(1)}, i=$ $1, \ldots, N-1)$ of the form

$$
\begin{aligned}
& \lim _{N \rightarrow \infty}\left(\frac{N}{\pi Q L}\right)^{N} \frac{(\pi Q L / N)^{\frac{N-1}{2}}}{\sqrt{N}} \times \\
& \int_{-\infty}^{\infty} \prod_{i=1}^{N-1} d u_{i}^{(1)} e^{-\frac{N}{Q L} \sum_{i, j=1}^{N-1} u_{i}^{(1)} M_{i, j}(\alpha) u_{j}^{(1)}}
\end{aligned}
$$

where we denote $\alpha=\frac{4}{N}\left(\frac{\mu}{N}\right)^{2}$, and the $(N-1)$ by $(N-1)$ matrix $M(\alpha)$ has the following elements: $M_{i, i}=2+\alpha$, $M_{i, i \pm 1}=-1+\alpha, i=1, \ldots, N-1, M_{i, j}=\alpha, j \neq i, j \neq$ $i \pm 1$. It is straightforward to calculate the determinant 
of $M(\alpha)$ and hence to perform the Gaussian integration over $u_{i}^{(1)}$

$$
\begin{gathered}
\operatorname{det}[M(\alpha)]=N+\alpha \frac{N^{2}\left(N^{2}-1\right)}{12}, \\
I_{Q C}^{(0)}\left[\Psi_{0}(z)\right]=\frac{1}{\pi Q L \sqrt{1+\mu^{2} / 3}} .
\end{gathered}
$$

To calculate the next-to-leading order contribution in $\sqrt{Q L}$ to the path-integral (B4) we should take the second term in the square brackets in Eq. (B4). To find this correction we should calculate the integral (the correlator):

$$
\begin{aligned}
& \left\langle u^{(\alpha)}(z) u^{(\beta)}\left(z^{\prime}\right)\right\rangle \equiv Q L G^{\alpha, \beta}\left(z, z^{\prime}\right) \equiv \frac{1}{I_{Q C}^{(0)}\left[\Psi_{0}(z)\right]} \times \\
& u(L)=0 \\
& \int_{u(0)=0} \mathcal{D} u e^{-\frac{1}{Q} \int_{0}^{L} d z\left|\partial_{z} u-i \frac{\mu}{L}(u+\bar{u})\right|^{2}} u^{(\alpha)}(z) u^{(\beta)}\left(z^{\prime}\right),(\mathrm{B} 11)
\end{aligned}
$$

where we have introduced the dimensionless Green matrix $G^{\alpha, \beta}\left(z, z^{\prime}\right), \alpha, \beta=1,2$. The standard method for the Green matrix calculation is the calculation of the generating functional [18]:

$$
\begin{gathered}
Z\left[J_{1}, J_{2}\right]=\int_{u(0)=0}^{u(L)=0} \mathcal{D} u \exp \left\{-\frac{1}{Q} \int_{0}^{L} d z\left|\partial_{z} u-i \frac{\mu}{L}(u+\bar{u})\right|^{2}+\right. \\
\left.\int_{0}^{L} d z\left(J_{1}(z) u^{(1)}(z)+J_{2}(z) u^{(2)}(z)\right)\right\}
\end{gathered}
$$

then any correlator can be derived from the variation of the $Z\left[J_{1}, J_{2}\right]$ over $J_{\alpha}$, for example

$$
\begin{aligned}
& \left\langle u^{(\alpha)}(z) u^{(\beta)}\left(z^{\prime}\right)\right\rangle=Q L G^{\alpha, \beta}\left(z, z^{\prime}\right)= \\
& \left.\frac{1}{Z\left[J_{1}, J_{2}\right]} \frac{\delta Z\left[J_{1}, J_{2}\right]}{\delta J_{\alpha}(z) \delta J_{\beta}\left(z^{\prime}\right)}\right|_{J_{1}=0, J_{2}=0}
\end{aligned}
$$

The calculation of the generating functional can be performed in the same way as the calculation of the normalization integral (B6): the integration over $u_{j}^{(2)}$ followed by the integration over $u_{j}^{(1)}$. The only new element in the calculation of the Gaussian integrals with the sources $J_{\alpha}$ is the inverse matrix $M(\alpha)_{i, j}^{-1}$ for $M(\alpha)_{i, j}=$ $\alpha+2 \delta_{i, j}-\delta_{i, j+1}-\delta_{i+1, j}$ defined herein above, see the text after Eq. (B8). The calculation is simple (after the observation that $\operatorname{det}[M(\alpha)] M(\alpha)_{i, j}^{-1}$ is linear in $\alpha$ ), and we only present the result

$$
\begin{aligned}
& M(\alpha)_{i, j}^{-1}=N\left[-\frac{\alpha N^{4}}{4 \operatorname{det}[M(\alpha)]} \frac{i}{N}\left(1-\frac{i}{N}\right) \times\right. \\
& \frac{j}{N}\left(1-\frac{j}{N}\right)+\frac{i}{N}\left(1-\frac{j}{N}\right) \theta(i \leq j)+ \\
& \left.\frac{j}{N}\left(1-\frac{i}{N}\right) \theta(i>j)\right],
\end{aligned}
$$

where $\operatorname{det}[M(\alpha)]$ is given by Eq. (B9), and $\lim _{N \rightarrow \infty}\left(\alpha N^{4} /(4 \operatorname{det}[M(\alpha)])\right)=3 \mu^{2} /\left(3+\mu^{2}\right)$. We present the result of the generating functional calculation in the form of a Green matrix convolution with the sources $J_{\alpha}$ :

$$
Z\left[J_{1}, J_{2}\right]=\frac{e^{\frac{Q L}{2} \int_{0}^{L} d z \int_{0}^{L} d z^{\prime} J_{\alpha}(z) G^{\alpha, \beta}\left(z, z^{\prime}\right) J_{\beta}\left(z^{\prime}\right)}}{\pi Q L \sqrt{1+\mu^{2} / 3}}
$$

where the Green matrix is Hermitian and it has the following elements:

$$
\begin{aligned}
& G^{1,1}\left(z, z^{\prime}\right)=G^{1,1}\left(z^{\prime}, z\right)=\left\{\theta\left(z^{\prime}-z\right) \frac{z}{2 L}\left(1-\frac{z^{\prime}}{L}\right)-\frac{3 \mu^{2}}{4\left(3+\mu^{2}\right)}\left(1-\frac{z}{L}\right)\left(1-\frac{z^{\prime}}{L}\right) \frac{z z^{\prime}}{L^{2}}\right\}+\left\{z \leftrightarrow z^{\prime}\right\} \\
& G^{1,2}\left(z, z^{\prime}\right)=G^{2,1}\left(z^{\prime}, z\right)=\frac{\mu}{2\left(3+\mu^{2}\right)}\left\{\theta\left(z-z^{\prime}\right) \frac{z^{\prime}}{L}\left(1-\frac{z}{L}\right)\left(3 \frac{z^{\prime}}{L}-3 \frac{z}{L}+\frac{z^{\prime}}{L} \mu^{2}\left[1+\frac{z}{L}\left(2 \frac{z^{\prime}}{L}-3\right)\right]\right)+\right. \\
& \left.\theta\left(z^{\prime}-z\right) \frac{z}{L}\left(1-\frac{z^{\prime}}{L}\right)\left(3 \frac{z^{\prime}}{L}-3 \frac{z}{L}+\left(\frac{z^{\prime}}{L}-1\right) \mu^{2}\left[\frac{z}{L}+2 \frac{z^{\prime}}{L}\left(\frac{z}{L}-1\right)\right]\right)\right\}
\end{aligned}
$$




$$
\begin{aligned}
& G^{2,2}\left(z, z^{\prime}\right)=G^{2,2}\left(z^{\prime}, z\right)=\left\{\frac { \theta ( z - z ^ { \prime } ) } { 6 ( 3 + \mu ^ { 2 } ) } ( 1 - \frac { z } { L } ) \frac { z ^ { \prime } } { L } \left[9+3 \mu^{2}\left(1+\frac{z}{L}-2 \frac{z^{2}}{L^{2}}+3 \frac{z z^{\prime}}{L^{2}}-2 \frac{z^{\prime 2}}{L^{2}}\right)+\right.\right. \\
& \left.\left.2 \mu^{4} \frac{z^{\prime}}{L}\left(\frac{z}{L}-1\right)\left(\frac{z^{\prime}}{L}-3 \frac{z}{L}+2 \frac{z z^{\prime}}{L^{2}}\right)\right]\right\}+\left\{z \leftrightarrow z^{\prime}\right\} .
\end{aligned}
$$

The second way to obtain the expression for the correlator (B11) and Eqs. (B16)-(B18) reflects the fact that the Gaussian integral (B12) is saturated in the vicinity of the saddle-point solution of the equation of motion (i.e. the Euler-Lagrange equation for the action in question) [19]. Thus to find it we should solve the set of equations

$$
\hat{K}_{\alpha, \gamma} G^{\gamma, \beta}\left(z, z^{\prime}\right)=\frac{1}{L} \delta_{\alpha}^{\beta} \delta\left(z-z^{\prime}\right)
$$

where the matrix differentiation operator $\hat{K}$ for the functions $u(z=0)=u(z=L)=0$ is defined as

$$
\begin{aligned}
& -\frac{1}{Q} \int_{0}^{L} d z\left|\partial_{z} u-i \frac{\mu}{L}(u+\bar{u})\right|^{2}= \\
& -\frac{1}{2 Q} \int_{0}^{L} d z u^{(\alpha)}(z) \hat{K}_{\alpha, \beta} u^{(\beta)}(z),
\end{aligned}
$$

and it has the form

$$
\hat{K}=2\left(\begin{array}{cc}
-\partial_{z}^{2}+\frac{4 \mu^{2}}{L^{2}}, & -2 \frac{\mu}{L} \partial_{z} \\
2 \frac{\mu}{L} \partial_{z}, & -\partial_{z}^{2}
\end{array}\right) .
$$

The boundary conditions for equations (B19) are as follows: $G^{\alpha, \beta}\left(z=0, z^{\prime}\right)=G^{\alpha, \beta}\left(z=L, z^{\prime}\right)=0$. The problem has the unambiguous solution (B16)-(B18). Note that the homogeneous solution of the Eq. (B19) is governed by the solutions of Eq. (A20) obtained above.

Using the correlator (B11) with (B16)-(B18) one can easily calculate the first correction presented in Eq. (B4). This term is proportional to $\varkappa_{1}(z) \propto \sqrt{Q L}$ hence delivering the leading correction to the leading term (B10). The subsequent integration of the elements (B16)-(B18) with the solution (A22) for $\varkappa_{1}(z)$ is trivial, however the proper way to understand the discontinuous derivatives of the Green matrix elements (B16)-(B18) at the same point $z^{\prime}=z$ is the retarded scheme adopted in our approach [17]: $\left.\partial_{z} G^{\alpha, \beta}\left(z, z^{\prime}\right)\right|_{z^{\prime}=z} \rightarrow \partial_{z} G^{\alpha, \beta}(z+$ $\left.0, z^{\prime}\right)\left.\right|_{z^{\prime}=z}$. Finally we have

$$
\begin{aligned}
& I_{Q C}\left[\Psi_{0}(z)\right]=\frac{1}{\pi Q L \sqrt{1+\mu^{2} / 3}}\left[1-\frac{\mu / \rho}{15\left(1+\mu^{2} / 3\right)^{2}} \times\right. \\
& \left.\left(\mu\left(15+\mu^{2}\right) x_{0}-2\left(5-\mu^{2} / 3\right) y_{0}\right)\right] .
\end{aligned}
$$

This result is obtained with the accuracy of the terms proportional to $Q L$. In Ref. [20] we found these corrections to the path-integral contribution as well.

Finally, from Eq. (A28) for the exponent factor and from Eq. (B22) for the pre-exponent factor we arrive at the expression

$$
\begin{aligned}
& P[Y \mid X]=\frac{\exp \left\{-\frac{\left(1+4 \mu^{2} / 3\right) x_{0}^{2}-2 \mu x_{0} y_{0}+y_{0}^{2}}{Q L\left(1+\mu^{2} / 3\right)}\right\}}{\pi Q L \sqrt{1+\mu^{2} / 3}}\left(1-\frac{\mu / \rho}{15\left(1+\mu^{2} / 3\right)^{2}}\left(\mu\left(15+\mu^{2}\right) x_{0}-2\left(5-\mu^{2} / 3\right) y_{0}\right)-\right. \\
& \frac{\mu / \rho}{135 Q L\left(1+\mu^{2} / 3\right)^{3}}\left\{\mu\left(4 \mu^{4}+15 \mu^{2}+225\right) x_{0}^{3}+\left(23 \mu^{4}+255 \mu^{2}-90\right) x_{0}^{2} y_{0}+\mu\left(20 \mu^{4}+117 \mu^{2}-45\right) x_{0} y_{0}^{2}-\right. \\
& \left.\left.3\left(5 \mu^{4}+33 \mu^{2}+30\right) y_{0}^{3}\right\}\right) .
\end{aligned}
$$

Now it is easy to show, that the normalization condition

$$
\int D Y P[Y \mid X]=1
$$

is fulfilled.

\section{Appendix C: CALCULATION OF $P_{\text {out }}[Y]$.}

Let us consider the integral $P_{\text {out }}[Y]=$ $\int \mathcal{D} X P_{X}[X] P[Y \mid X]$. In our case the measure $\mathcal{D} X=d x d y$, where $x=\operatorname{Re}\{X\}, y=\operatorname{Im}\{X\}$, so 
we should consider the integral:

$$
\int_{-\infty}^{\infty} d x d y P_{X}[x, y] P[Y \mid X]
$$

In the integral the scale of variation of the function $P_{X}[x, y]$ is $P \gg Q L$. The scale of variation of the function $P[Y \mid X]$ is $Q L$, and this function has the form Eq. (22), therefore we can use Laplace's method. To demonstrate that one can see that the function $P[Y \mid X]$ depends on $|X|, x_{0}=\operatorname{Re}\left\{\bar{X}\left(Y e^{-i \mu}-X\right) /|X|\right\}, y_{0}=$ $\operatorname{Im}\left\{\bar{X}\left(Y e^{-i \mu}-X\right) /|X|\right\}$, and reaches the maximal value at the point $x_{0}=y_{0}=0$. Let us change the integration variables $x, y$ to $\eta_{1}, \eta_{2}$, where $\eta=\eta_{1}+i \eta_{2}=\left(X e^{i \mu}-\right.$ $Y) e^{-i \phi^{(Y)}}$. Here $\phi^{(Y)}$ is the phase of the $Y$. The inverse transformation reads $X=(\eta+|Y|) e^{-i \gamma L|\eta+| Y||^{2}+i \phi^{(Y)}}$. In the new variables the function $P[Y \mid X]$ reaches maximum at the point $\eta_{1}=\eta_{2}=0$. The integral (C1) takes the following form

$$
\int_{-\infty}^{\infty} d \eta_{1} d \eta_{2} P[Y \mid X] P_{X}\left[\operatorname{Re}\left\{(\eta+|Y|) e^{-i \gamma L|\eta+| Y||^{2}+i \phi^{(Y)}}\right\}, \operatorname{Im}\left\{(\eta+|Y|) e^{-i \gamma L|\eta+| Y||^{2}+i \phi^{(Y)}}\right\}\right]
$$

here we have used the fact that the Jacobian determinant for the variables transformation is equal to unity. Since $P[Y \mid X]$ reaches its maximum at the point $\eta=0$ we can expand the functions $P_{X}[X]$ and $P[Y \mid X]$ in the vicinity of the point:

$$
\begin{aligned}
& P_{X}\left[\operatorname{Re}\left\{(\eta+|Y|) e^{-i \gamma L|\eta+| Y||^{2}+i \phi^{(Y)}}\right\}, \operatorname{Im}\left\{(\eta+|Y|) e^{-i \gamma L|\eta+| Y||^{2}+i \phi^{(Y)}}\right\}\right] \approx \\
& \quad\left(P_{X}\left[\operatorname{Re}\left\{Y e^{-i \tilde{\mu}}\right\}, \operatorname{Im}\left\{Y e^{-i \tilde{\mu}}\right\}\right]+\text { terms proportional to } \eta+\ldots\right) \\
& P[Y \mid X] \approx \frac{1}{\pi Q L \sqrt{1+\tilde{\mu}^{2} / 3}} \exp \left\{-\frac{\left(1+4 \tilde{\mu}^{2} / 3\right) \eta_{1}^{2}-2 \tilde{\mu} \eta_{1} \eta_{2}+\eta_{2}^{2}}{Q L\left(1+\tilde{\mu}^{2} / 3\right)}\right\}\left(1+\text { terms proportional to } \eta \text { and } \frac{\eta^{3}}{Q L}\right)
\end{aligned}
$$

where we have used the fact that in the vicinity of the point $\eta=0$ we have $x_{0}=-\eta_{1}$ and $y_{0}=-\eta_{2}$ up to higher powers of $\eta$. In Eqs. (C3) and (C4) we have the parameter $\tilde{\mu}=\gamma L|Y|^{2}$.

One can see that at large $\tilde{\mu}$ the exponent contains three different terms:

$$
\begin{aligned}
& \frac{\left(1+4 \tilde{\mu}^{2} / 3\right) \eta_{1}^{2}-2 \tilde{\mu} \eta_{1} \eta_{2}+\eta_{2}^{2}}{Q L\left(1+\tilde{\mu}^{2} / 3\right)} \approx \\
& \frac{4 \eta_{1}^{2}}{Q L}-6 \frac{\eta_{1} \eta_{2}}{Q L \tilde{\mu}}+\frac{3 \eta_{2}^{2}-9 \eta_{1}^{2}}{Q L \tilde{\mu}^{2}} .
\end{aligned}
$$

Therefore to use Laplace's method we have to transform our quadratic form

$$
\left(\eta_{1}, \eta_{2}\right) A\left(\eta_{1}, \eta_{2}\right)^{T}=\frac{\left(1+4 \tilde{\mu}^{2} / 3\right) \eta_{1}^{2}-2 \tilde{\mu} \eta_{1} \eta_{2}+\eta_{2}^{2}}{Q L\left(1+\tilde{\mu}^{2} / 3\right)}
$$

to the canonical form. The matrix of quadratic form is:

$$
A=\frac{1}{Q L\left(1+\tilde{\mu}^{2} / 3\right)}\left(\begin{array}{cc}
1+\frac{4 \tilde{\mu}^{2}}{3} & -\tilde{\mu} \\
-\tilde{\mu} & 1
\end{array}\right) .
$$

The eigenvalues of the matrix $A$ are

$$
\begin{aligned}
& \lambda_{1}=\frac{1}{Q L}\left(1+\tilde{\mu} \frac{\tilde{\mu}+\sqrt{9+4 \tilde{\mu}^{2}}}{3+\tilde{\mu}^{2}}\right), \\
& \lambda_{2}=\frac{1}{Q L}\left(1+\tilde{\mu} \frac{\tilde{\mu}-\sqrt{9+4 \tilde{\mu}^{2}}}{3+\tilde{\mu}^{2}}\right) .
\end{aligned}
$$

One can see that $\lambda_{1,2}>0$, and at large $\tilde{\mu}$ they have the form:

$$
\lambda_{1} \approx \frac{4}{Q L}, \quad \lambda_{2} \approx \frac{3}{4 Q L \tilde{\mu}^{2}} .
$$

Therefore at large $\mu \approx \tilde{\mu}$ there are two parameters in the Laplace integral, one parameter is $1 / Q L$, the other is $1 /\left(Q L \tilde{\mu}^{2}\right)$. To use Laplace's method for the integral Eq. (C1) we have to impose two conditions $P \gg Q L$, and $P \gg Q L \tilde{\mu}^{2}$. These conditions lead to the two dimensionless parameters for Laplace's method

$$
\begin{aligned}
& \mathrm{SNR} \gg 1, \\
& \left(\gamma^{2} Q L^{3} P\right)^{-1} \gg 1 .
\end{aligned}
$$

To calculate the integral Eq. $(\mathrm{C} 2)$ in the leading order in the parameters $1 / \mathrm{SNR}$ and $\left(\gamma^{2} Q L^{3} P\right)$ we substitute the first term of the expansion Eq. (C3) and the first term in the brackets of the expansion Eq. (C4) to the integral Eq. (C1). After straightforward calculation we obtain:

$$
\begin{aligned}
& P_{X}\left[\operatorname{Re}\left\{Y e^{-i \gamma L|Y|^{2}}\right\}, \operatorname{Im}\left\{Y e^{-i \gamma L|Y|^{2}}\right\}\right] \times \\
& \int_{-\infty}^{\infty} d \eta_{1} d \eta_{2} P[Y \mid X] \approx P_{X}\left[Y e^{-i \gamma L|Y|^{2}}\right] .
\end{aligned}
$$

To calculate corrections to the integral in parameters $1 / \mathrm{SNR}$ and $\gamma^{2} Q L^{3} P$ we should take terms which are proportional to $\eta$ and $\eta^{3}$ in the product of expansions Eqs. 
(C3) and (C4). Formally the first correction to the integral should be of order of $1 / \sqrt{\mathrm{SNR}}$ and $\sqrt{\gamma^{2} Q L^{3} P}$, but it is zero due to the symmetry $\eta \rightarrow-\eta$ (the exponent contains only even combination of $\eta$ ). Therefore up to terms proportional to $Q L$ the result for the integral Eq. (C1) has the form

$$
\int_{-\infty}^{\infty} d x d y P_{X}[x, y] P[Y \mid X] \approx P_{X}\left[Y e^{-i \gamma L|Y|^{2}}\right] .
$$

[1] C. Shannon, Bell Syst. Tech. J. 27, 379 (1948); 27, 623 (1948).

[2] P. Mitra and J. B. Stark, Nature 411, 1027 (2001).

[3] E. E. Narimanov and P. Mitra, J. Lightwave Technol. 20, 530 (2002).

[4] J. M. Kahn and K.-P. Ho, IEEE. J. Sel. Topics Quant. Electron. 10, 259 (2004)

[5] R.-J. Essiambre, G. J. Foschini, G. Kramer, and P. J. Winzer, Phys. Rev. Lett. 101, 163901 (2008).

[6] R.-J. Essiambre, G. Kramer, P. J.Winzer, G. J. Foschini, and B. Goebel, J. Lightwave Technol. 28, 662 (2010).

[7] R. Killey and C. Behrens, J. Mod. Opt. 58, 1 (2011).

[8] E. Agrell, A. Alvarado, G. Durisi, and M. Karlsson, IEEE/OSA J. Lightwave Technol. 32, 2862 (2014).

[9] M. A. Sorokina and S. K. Turitsyn, Nat. Commun. 5, 3861 (2014).

[10] A. Mecozzi, J. Lightwave Technol. 12, 1993 (1994).

[11] A. Mecozzi and M. Shtaif, IEEE Photonics Technol. Lett. 13, 1029 (2001)

[12] J. Tang, J. Lightwave Technol. 19, 1104 (2001).

[13] K. S. Turitsyn, S. A. Derevyanko, I. V. Yurkevich, and S. K. Turitsyn, Phys. Rev. Lett. 91, 203901 (2003).

[14] M. I. Yousefi and F. R. Kschischang, IEEE Trans. Inf.
Theory 57, 7522 (2011).

[15] E. Agrell, IEEE Trans. Commun. 63, 738 (2015).

[16] E. Agrell, in Proceedings of the 39th European Conference and Exhibition on Optical Communication (ECOC 2013) (IEEE/IET Electronic Library, New York, 2013), pp. 13.

[17] I. S. Terekhov, S. S. Vergeles, and S. K. Turitsyn, Phys. Rev. Lett. 113, 230602 (2014).

[18] J. Zinn-Justin, Quantum Field Theory and Critical Phenomena (Oxford University Press, Oxford, UK, 2002).

[19] R. P. Feynman and A. R. Hibbs, Quantum Mechanics and Path Integrals (McGraw-Hill, New York, 1965).

[20] A. A. Panarin, A. V. Reznichenko, I. S. Terekhov, Phys. Rev. E 95, 012127 (2017).

[21] M. A. Lavrentiev and B.V. Shabat, Method of Complex Function Theory (Nauka, Moscow, 1987); M. Lavrentiev and B. Chabot, Methodes de la Theorie des fonctions dune variable complexe (Mir, Moscow, 1977).

[22] I. S. Gradshtein and I. M. Ryzik, Table of Integrals and Series, and Products (Academic Press, Orlando, Florida, 2014). 